\title{
Similarity, typicality, and category-level matching of morphed outlines of everyday objects
}

\author{
Sven Panis, Joris Vangeneugden, Johan Wagemans $\uparrow$ \\ Laboratory of Experimental Psychology, University of Leuven, Tiensestraat 102, B 3000 Leuven, Bel- \\ gium; e-mail: johan.wagemans@psy.kuleuven.be \\ Received 4 October 2007, in revised form 3 July 2008; published online 10 December 2008
}

\begin{abstract}
During visual object categorisation, a match must be found between the input image and stored information about basic-level categories. Graf [2002 Form, Space and Object (Berlin: Wissenschaftlicher Verlag Berlin)] suggested the involvement of analogue transformational, shapechanging processes in aligning the memory representation of the category with the perceptual representation of the current stimulus. Here we compare the predictions of alignment models with those of exemplar-based models, using morphing between four exemplar outlines within each of eleven categories. Overall, with increasing transformational distance between two exemplars of the same category, reaction times to decide whether they belong to the same category in a sequential matching paradigm increased, while rated similarity between the two exemplars decreased. However, in contrast to alignment accounts, exemplar-based accounts can correctly predict the observed dissociation between typicality and categorisation time, and allow the observed deviations from sequential additivity and nonlinear relations between transformational distance and rated similarity. Discussion of integrations of exemplar-based theories with neglected processes, such as information accumulation, response competition, response priming, and gain-modulation leads to a view of the recognition process from input to response, which increases the validity and scope of modern exemplar-based categorisation and recognition models.
\end{abstract}

\section{Introduction}

Retinal images of the same object can be highly variable because of different lightingand viewing-conditions. Nevertheless, humans can recognise a familiar object despite spatial transformations such as translations or rotations in the picture-plane, changes in the size of its image, or even rotations in depth. Similarly, retinal images of different objects from the same category are highly variable because of additional changes in shape, colour, texture, etc. Again, though, humans can categorise objects which they have never seen before as belonging to a certain basic-level class (eg a car, a chair, a bird, a dog, etc) using the similarity of the image with stored class information (for reviews, see Mervis and Rosch 1981; Palmeri and Gauthier 2004). Categorisation may be a useful intermediate stage on the way to more specific identification, because it restricts the set of candidate object models and because the use of class-specific information allows a broader generalisation (Ullman 1989, 1998). However, it is not yet clear how the visual system deals with the large range of possible images when classifying and identifying objects.

One observation that has attracted a lot of attention is the behavioural dependence on these image transformations. Behavioural studies, in which two images of the same object are presented sequentially and subjects have to decide whether body images belong to the same object or not, have revealed a monotonic increase in reaction times (RTs) and/or error rates with increasing transformational distance between the two images. This behavioural dependence on spatial transformations has been observed for translations, size-scalings, rotations in the picture-plane and in depth (Bundesen et al 1981, 1983; Larsen and Bundesen 1978; Larsen et al 1999; Lawson 1999; Shepard and Cooper 1982), and recently also for shape-changing or topological transformations (Graf 2002, 2006). 
Graf (2002, 2006, 2008) proposed a transformational model of recognition and categorisation (TMRC) based on a process-based interpretation of Felix Klein's Erlanger Programm. ${ }^{(1)}$ By incorporating the general class of shape-changing or topological transformations (which includes also, as a result of the hierarchies between the geometries, all lower-level transformations such as affine and Euclidean transformations), alignment models for recognition can be extended to account for categorisation performance. Recognition after spatial transformations relies mostly on Euclidean transformation processes, while categorisation up to the basic level can be accounted for mostly by non-Euclidean topological transformations. Thus, categorisation and recognition are achieved by transforming (the coordinates of) a generic perceptual coordinate system or reference frame that defines the correspondence between positions specified in memory and positions in the current visual field so that the category and object representations in memory and the input representation can be more or less aligned, to compensate for the image transformation.

TMRC explains the behavioural dependence on image transformations (and other findings) parsimoniously by hypothesising that the transformations of the coordinates of the perceptual reference frame are fast (in contrast to slower image transformations during mental rotation), analogue (ie they traverse intermediate points along the transformational path), and implemented by neural gain (amplitude) modulation (Salinas and Sejnowski 2001). Evidence for the analogue nature of transformational processes comes from a study by Bundesen et al (1981) who showed that RTs to decide if two sequentially presented shapes are identical except for changes in picture-plane orientation or size, were sequentially additive, suggesting analogue rotation- and size-scaling processes in object recognition. Evidence for the involvement of a reference frame comes from a study by Graf et al (2005) that revealed orientation-congruency effects between two familiar shapes of different basic-level and superordinate-level categories (see also Jolicoeur 1990): higher naming accuracy was found when both sequentially presented object images were displayed in congruent instead of incongruent picture-plane orientations. Finally, according to TMRC, the typicality of a stimulus for a certain category is based on the amount of topological transformation which is necessary to align the stimulus representation with the representation of the category prototype, while similarity between two stimuli is based on the transformational distance between both stimuli. This latter idea is supported by studies suggesting that the similarity between two objects - a concept at the core of any categorisation theory-is based on the transformational distance between the structural representations of those objects (Hahn et al 2003; Markman and Gentner 1993a, 1993b).

Graf (2002) tested his transformational model by studying the dependence of similarity and typicality judgments and sequential-matching performance on topological transformations, using outlines of category exemplars and warping software (see also Graf 2008). For each of twenty-two categories, he selected two exemplars and created one or three new exemplars that were positioned at a specific transformational distance between the selected ones in the warning sequence. As predicted by TMRC, Graf found that RTs and errors increased monotonically with increasing topological distance between two exemplars when subjects had to indicate whether both exemplars belong to the same basic-level category or not, while rated similarity decreased monotonically. Furthermore, the categorisation latencies did not seem to be incompatible with the hypothesis of sequential additivity, which was taken to be suggestive of analogue transformational processes. Finally, typicality ratings usually decreased monotonically

(1) In his well-known Erlanger Programm, Felix Klein has established a hierarchy of progressively weaker geometries, consisting of groups of transformations with fewer and broader invariants, which give rise to progressively wider equivalence classes: from Euclidean, to similarity and affine, to projective geometry, and topology (Todd et al 1998; Van Gool et al 1994; Wagemans et al 1997). 
with increasing distance from the position of the exemplar that was rated as most typical; the latter presumably being closest to the representation of the category prototype, conceptualised as a superposition of topologically transformed images of category exemplars. The data suggested that there was one prototypical exemplar for natural categories, while there were two for artifactual categories.

Graf's TMRC is interesting because it treats recognition and categorisation within a unified framework, integrating a wide variety of findings. However, it is also confronted with a number of problems. First, the warping procedure that is used does indeed imply a change in object shape that is more than Euclidean, affine, or projective, but it does not establish a well-defined topological transformation that is easily parameterised. While shape matching under Euclidean transformations (planar translations and rotations), similarities (uniform size-scalings), affine (nonuniform size-scalings and shears), and projective transformations has been studied extensively (Bundesen et al 1981, 1983; Demeyer et al 2007; Kukkonen et al 1996; Larsen and Bundesen 1978; Larsen et al 1999; Tarr 1995; Tarr et al 1998; Vanrie et al 2001, 2002; Wagemans et al 1996, 1997, 2000; Willems and Wagemans 2001), the role of topology for visual perception is less evident. Chen $(1985,2005)$ has suggested that topological transformations and the properties they leave invariant (connectedness, inside-outside relations, the presence of holes) are useful for understanding perceptual organisation but that does not mean that the human visual system implements topological transformations as such. The only evidence so far has come from research on the perception of growth or aging (Mark and Todd 1985; Mark et al 1981; Pittenger and Shaw 1975; Pittenger and Todd 1983), which was inspired by theoretical work in biology by D'Arcy Thompson (1917/1942).

Second, it cannot be ruled out that subjects are sensitive to the invariant properties preserved under each of these transformations (for an introduction to the mathematical framework of transformations and invariants, see Van Gool et al 1994). As mathematical entities, invariants are-by definition-invariant and hence independent of the parameters of the transformation under which they are invariant. However, the way in which the visual system extracts or computes these invariant properties may be influenced quite systematically by the parameter values specifying the size of the transformation, which may give rise to the same systematic effects on performance measures (Wagemans et al 1996).

Finally, with his notion of abstract category representations, Graf (2002, 2006) appears to be swimming against the tide. During the last decades, the object-recognition and categorisation literatures have been moving away from abstract category representations to shift their focus more towards image-based exemplar representations that are size-, orientation-, view-, and shape-specific (Gauthier and Palmeri 2002; Kruschke 1992; Medin and Schaffer 1978; Mozer 2002; Nosofsky and Zaki 2002; Op de Beeck et al 2001, 2003, 2008; Palmeri and Gauthier 2004; Perrett et al 1998; Poggio and Bizzi 2004; Tarr and Bülthoff 1998; Vogels 1999; Zaki et al 2003). According to exemplarbased theories, objects are represented as collections of viewpoint-dependent features (eg the output of receptive fields) that are present in the retinal images of the objects. Each view can then be considered a point in a high-dimensional space that captures the appearance of all possible views. Recognising (or categorising) unknown object views (or category exemplars) is based on establishing the detailed (or coarse) location of the unknown view (or exemplar) within this space, by measuring the similarity of its features relative to the features of the nearest known views (or exemplars) in memory, known as interpolating across the view space (or interpolation to multiple visually similar exemplars, respectively_Edelman 1998; Tarr and Bülthoff 1998). These view-based or exemplar-based representations are able to sustain class-based generalisation without the need to assume the existence of compensation processes such as alignment (Tarr and Gauthier 1998). 
In most mathematically formulated exemplar-based models, a shape is represented by its similarity to stored prototypical exemplars from different categories, while similarity is a decreasing function of the distance between the input and the stored exemplars in a low-dimensional psychological shape space (Edelman 1998, 1999; Nosofsky 1986). Selective attention to certain dimensions systematically modifies the structure of the space in which the exemplars are embedded, making similarity context-dependent (Nosofsky 1986). Classification decisions are based on the similarity of a probe to exemplars of a target category relative to exemplars of contrast categories; recognition decisions are based on the overall summed similarity of a probe to all exemplars; and typicality judgments are governed by summed similarity of a probe to all exemplars of the target category (Nosofsky 1988).

These arguments raise doubts about the proposal that topological transformations are used to match images of different exemplars of a basic-level category to a category prototype (Graf 2002, 2006, 2008). In light of its potential importance, this proposal is clearly in need of further and stronger empirical support.

The purpose of our study was two-fold. First, we wanted to create and validate a new stimulus set in which the shape-similarity between exemplars from the same category is systematically manipulated, to study the representation of shape-similarity at the neuronal level (Panis et al 2008b), or to study the effects of categorisation learning on the neural representation of shape similarity (Gillebert et al 2008). Second, we wanted to compare the predictions of Graf's TMRC with those from pure exemplarbased models using this new stimulus set, because we believe that it allows remediation for possibly important shortcomings in Graf's experiments (see section 2.1). In four experiments we collected similarity and typicality ratings, and measured the performance in a sequential basic-level matching task.

As explained below, several aspects of our results favour an exemplar-based account of categorisation over a transformational account. However, we will argue that modern exemplar-based categorisation models need to incorporate modeling of well-established but neglected processes such as temporal integration of information, gain-modulation, response competition, and response priming in order to account for experimental data obtained in a large range of visual tasks.

\section{Experiment 1. Construction of a new stimulus set}

\subsection{Introduction}

To test TMRC, Graf selected two exemplars per category between which to warp, by hand. As a result, it is possible that he selected images that were both fairly (proto)typical which might result in a large sensitivity to the single topological transformation that was subsequently tested for each category. In contrast, for each of eleven categories, we wanted to create a larger number of morphing sequences per category, and to select a larger number of exemplars more objectively by collecting similarity ratings for all the category exemplars from a published set of line drawings (Op de Beeck and Wagemans 2001; see also Op de Beeck et al 2000). Within each category, we then identified the four most dissimilar exemplars from a two-dimensional multi-dimensional scaling (MDS) solution. As we will show, this selection procedure resulted in selections of typical and atypical exemplars from the same category to morph between.

\subsection{Methods}

2.2.1 Participants. Thirty high-school student volunteers (mean age 17 years) participated in experiment 1 . They were tested in two sessions that lasted around $1 \mathrm{~h}$ each. In this and subsequent experiments, all participants were naive regarding the purpose of the experiment in which they participated, all were unfamiliar with the stimuli, and all had normal or corrected-to-normal vision. No one participated in more than one experiment. 
2.2.2 Stimuli. A published stimulus set of line drawings of 269 exemplars from twenty-five different categories (Op de Beeck and Wagemans 2001) was changed in two ways. First, we created silhouette and outline versions using a procedure which we have also applied to the set of line drawings by Snodgrass and Vanderwart (1980; see Wagemans et al 2008), because this allows for more experimental control over the relevant stimulus features (see also De Winter and Wagemans 2004). Second, eight categories were deleted either because (i) the edge-extraction algorithm failed to yield smoothly curved, closer contours, (ii) the exemplar outlines were not recognisable anymore, or (iii) the exemplar outlines were too similar. 180 exemplars from seventeen categories remained.

2.2.3 Procedure. Similarity ratings were collected for all the possible intra-categorical combinations of 2 exemplars $[N=1928$; one category with 7 exemplars, six with 10 exemplars, seven with 11 exemplars, and three with 12 exemplars giving $49+(6 \times 100)+$ $(7 \times 121)+(3 \times 144)=1928$ combinations]. Each subject was tested in two sessions separated by at least $24 \mathrm{~h}$ (eight randomly chosen categories in the first, nine in the second session). Ten subjects were tested with all the line drawings, ten with all the silhouettes, and ten with all the outlines. Each experimental trial started with a fixation cross for $500 \mathrm{~ms}$ followed by both stimulus presentations for $200 \mathrm{~ms}$ separated by an interstimulus interval of $500 \mathrm{~ms}$. Subjects had to rate the similarity between the two consecutive stimuli on a 6-point scale (1 very dissimilar; 6 very similar). A chin-rest was placed $57 \mathrm{~cm}$ away from the screen. Stimuli $(400 \times 300$ bitmaps) were shown on a 21 -inch monitor $(1024 \times 768$ pixels, $60 \mathrm{~Hz}$ refresh, background luminance $20 \mathrm{~cd} \mathrm{~m}^{-2}$ ) and subtended $14.2 \mathrm{deg} \times 10.9 \mathrm{deg}$. As in Graf (2002), subjects were made familiar with the shape differences between all the exemplars from each category (in the particular version they were tested with) before the experimental trials of that category started. For each category, they were shown one sheet of A4 paper with all exemplars in a random order.

\subsection{Results}

After applying the multi-dimensional scaling technique (MDS) on the similarity ratings from each category, eleven categories (airplane, beetle, bird, butterfly, car, chair, fish, guitar, motorcycle, rodent, and vase) survived the following two selection criteria. First, only categories for which a two-dimensional MDS solution was appropriate for the contour versions were selected (the criterion was that the screenplot showed a knee at two dimensions with a d-hat stress value - a measure of badness of fitof less than 0.1 for two dimensions). Second, only categories were selected for which the same 4 exemplars turned out as most dissimilar (ie were located at the extremes of each dimension) across the line drawings, silhouette, and outline versions. These four 'extreme' exemplars were selected so that they formed the largest and best possible rectangle in the 2-D shape space.

In figure 1 we present the MDS solutions of the original outline versions of four example categories and the 4 selected 'extreme' exemplars. Next, the commercially available Magic Morph software was used to create 33\% and 66\% morphs between each pair of the selected extreme exemplars from each category in the outline version. This resulted in six morphing sequences with four positions $(0-33-66-100)$ and 16 exemplar outlines for each of eleven categories (see figure 2 first column). Note that each of the selected original exemplars (figure 1) was used as a start point ('source') or end-point ('target') of three morphing sequences (figure 2).

Interestingly, for all categories at least one original exemplar was lying in a relatively isolated region (see figure 1). For each of the original exemplars, the average distance to the other original exemplars was used as a measure of 'isolatedness'. 

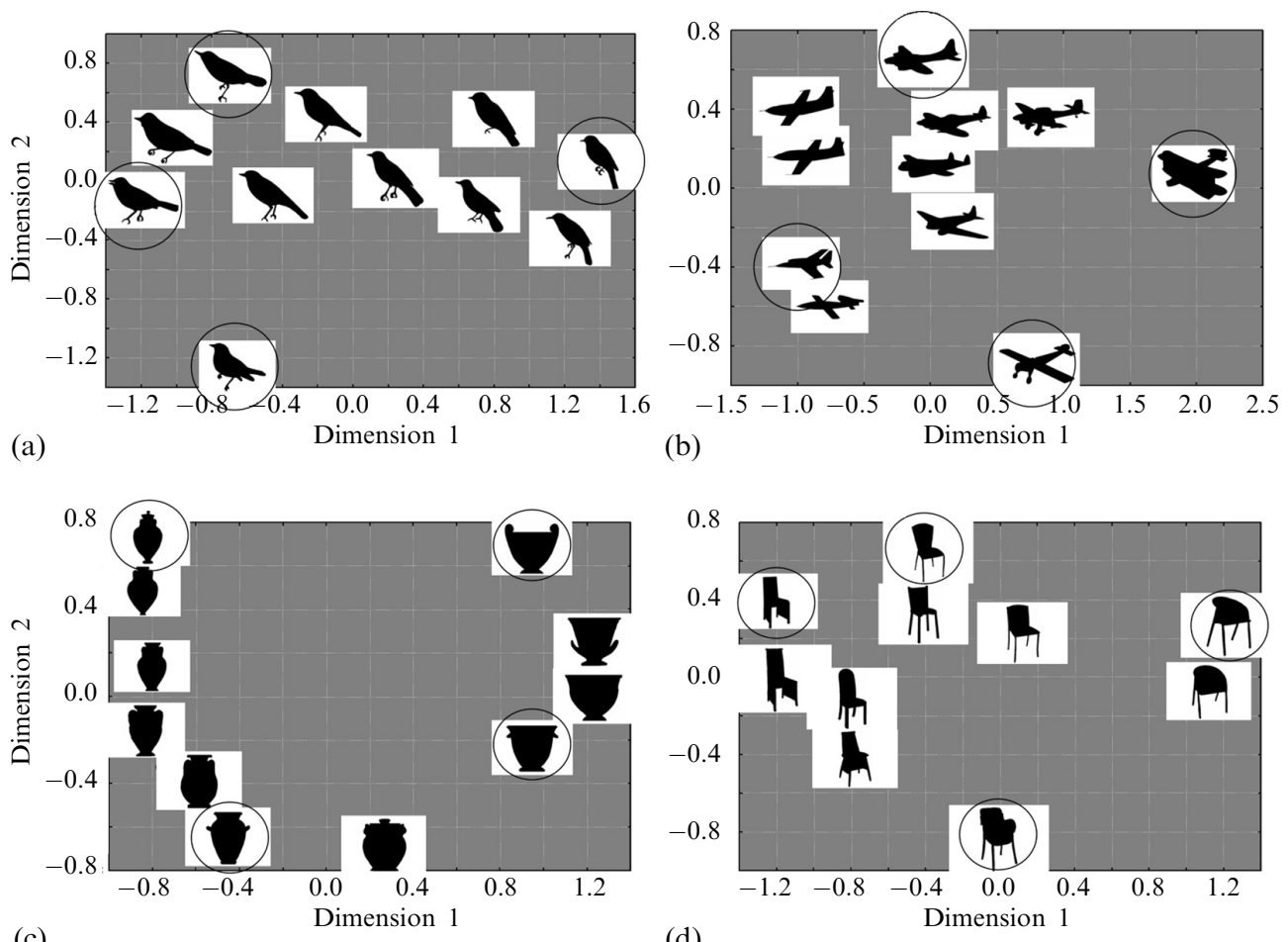

(c)

Figure 1. Two-dimensional solutions by multi-dimensional scaling (MDS) for four example categories. Circles are drawn around the 4 selected exemplars used to create morphing sequences (see figure 3): (a) birds; (b) airplanes; (c) vases; (d) chairs. Although the MDS solutions for the outlines are shown, the shapes are drawn as silhouettes for clarity.

All original exemplars per category were ranked from 1 to $7,10,11$, or 12 (depending on the category), from most to least isolated. The numbers in the four original exemplars used to create the morphs in figure 2 report these rank numbers. For nine out of eleven categories, the original exemplar with rank 1 was one of the 4 selected ones.

\subsection{Discussion}

To compare the predictions of TMRC and exemplar-based models regarding the effect of shape-changing transformations on similarity and typicality judgments and sequential matching performance in the next three experiments, we created 6 morphing sequences with 16 unique exemplar outlines for each of eleven categories. Because other researchers might be interested in using these stimuli, we made them available on our website (http://ppw.kuleuven.be/labexppsy/johanw/wag_2D.htm).

Although two-dimensional MDS solutions provided a reasonable fit to the data of the selected categories, the nature of the shape changes differed between the morphing sequences within each category. For example, the shape changes were small for the category guitar, and large for the categories fish and beetle. We used MDS only as a selection tool, and do not assume a true two-dimensional underlying physical or psychological space. Also, the presence of extreme exemplars in an isolated region offers an opportunity to test another prediction of an exemplar-based model (see experiment 3). 
20

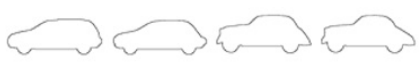

$\longrightarrow \longrightarrow$

(3)

$33 \Omega_{3}^{3} \Omega_{3}$

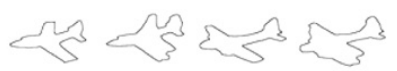

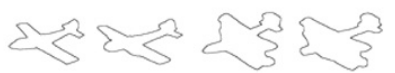

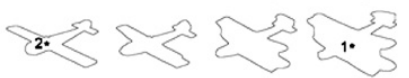
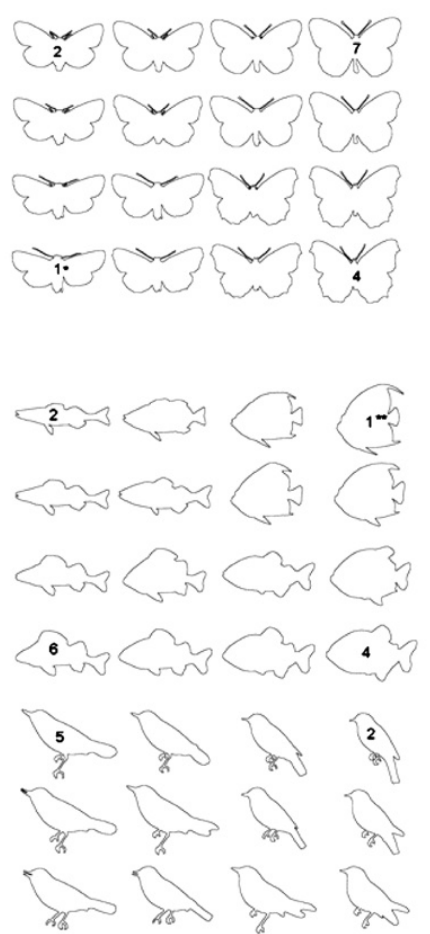

$P_{n \rightarrow \infty} \rightarrow R_{\text {and }}$

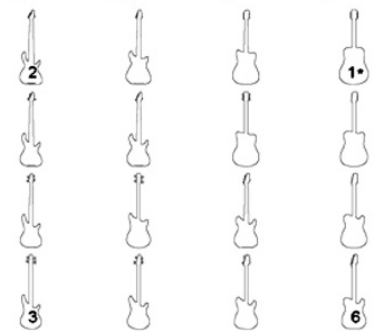

Figure 2 (see next page for caption)
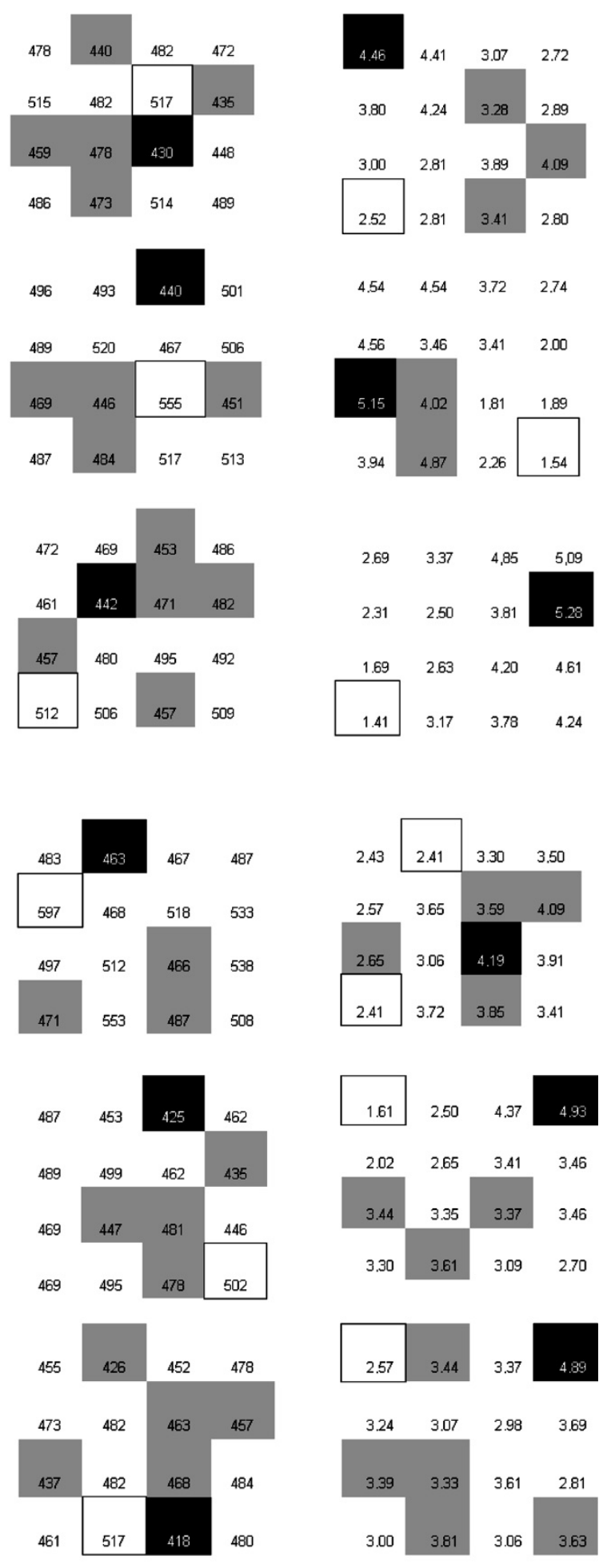

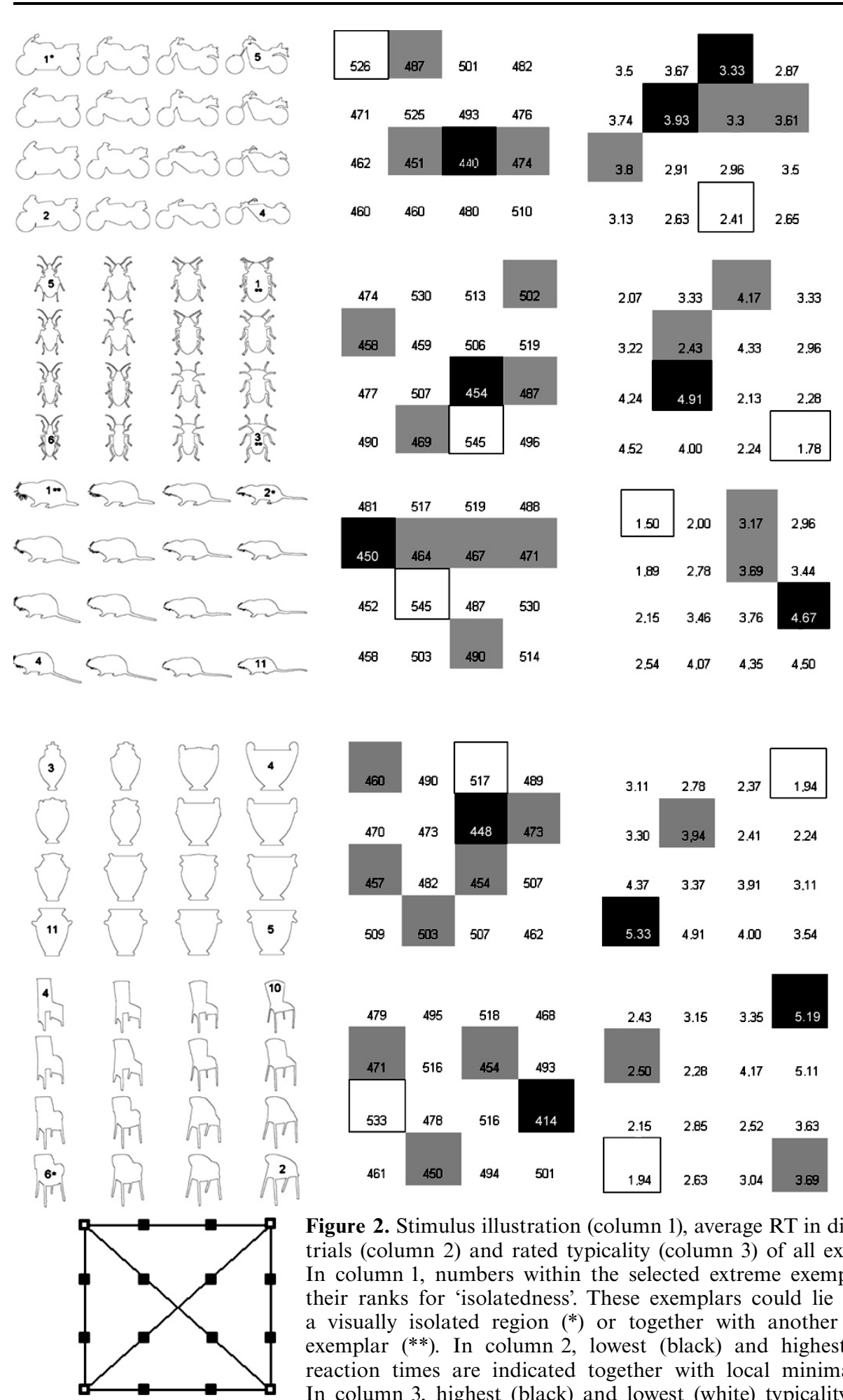

Figure 2. Stimulus illustration (column 1), average RT in distance-0 trials (column 2) and rated typicality (column 3) of all exemplars. In column 1, numbers within the selected extreme exemplars are their ranks for 'isolatedness'. These exemplars could lie alone in a visually isolated region $\left(^{*}\right)$ or together with another original exemplar (**). In column 2, lowest (black) and highest (white) reaction times are indicated together with local minima (grey). In column 3, highest (black) and lowest (white) typicality ratings are indicated together with local maxima (grey). The inset at the bottom shows the six morphing sequences and the four extreme (open squares) and 12 morphed exemplars (solid squares). Thus, both central horizontal and both central vertical lines do not correspond to a morphing sequence. 


\section{Experiment 2. Similarity and topological transformations}

\subsection{Introduction}

In experiment 2, we asked participants to rate the similarity for pairs of exemplars while we manipulated the transformational distance between them. According to TMRC, the similarity comparison is a two-stage process: the similarity of two shapes is influenced by the alignment phase, but also by the matching itself. If exact alignment is not achieved, remaining small shape differences are captured in the matching process (Graf 2002). TMRC and the transformational account of similarity (Hahn et al 2003) predict a monotonic decrease in rated similarity with increasing transformational distance between two outlines of the same category. In contrast, according to most exemplar-based theories, similarity is a decreasing function of the distance between the two points representing the object outlines in an underlying psychological space, which can differ between subjects.

\subsection{Methods}

3.2.1 Participants. Twenty high-school students participated voluntarily (mean age 17 years) and were tested individually in a single session that lasted around $45 \mathrm{~min}$.

3.2.2 Procedure. Each participant received 11 blocks of 96 trials. In each block, all the combinations between the 4 exemplars from each of the six morph sequences of one category were presented in separate trials $(4 \times 4 \times 6=96)$. As a result, 16 trial types were created: four distance-0 trials $(11,22,33,44)$, six distance- 1 or $33 \%$ trials $(12,21$, $23,32,34,43)$, four distance- 2 or $66 \%$ trials $(13,31,24,42)$, and two distance-3 or $100 \%$ trials $(14,41)$. The order of blocks (or categories) was randomised. Trials were self-paced and started with $500 \mathrm{~ms}$ fixation, followed by the sequential presentation of both stimuli for $150 \mathrm{~ms}$ each, separated by an interstimulus interval of $500 \mathrm{~ms}$. Subjects had to rate their similarity on a 6-point scale (1 very dissimilar; 6 very similar) after they were made familiar with the shape differences between all the exemplars from each category before each block of experimental trials started (by showing all of them on a single A4 page). Subjects were instructed not to think too long about their decision. A chin-rest was placed $57 \mathrm{~cm}$ away from the screen. Stimuli $(400 \times 300$ bitmaps) were shown centred on a 21 -inch monitor $(1024 \times 768$ pixels, $60 \mathrm{~Hz}$ refresh, background luminance $30 \mathrm{~cd} \mathrm{~m}^{-2}$ ) and subtended $14.2 \mathrm{deg} \times 10.9 \mathrm{deg}$.

\subsection{Results}

First, we calculated the average similarity ratings for each combination of subject and transformational distance (averages across categories, morphing sequences, and 2 to 6 trials with equal distance). A one-way ANOVA showed a significant effect of distance $\left(F_{3,57}=1412.73, p<0.0001\right)$. The average similarity ratings decreased with increasing transformational distance (figure 3 ). This was confirmed by a highly

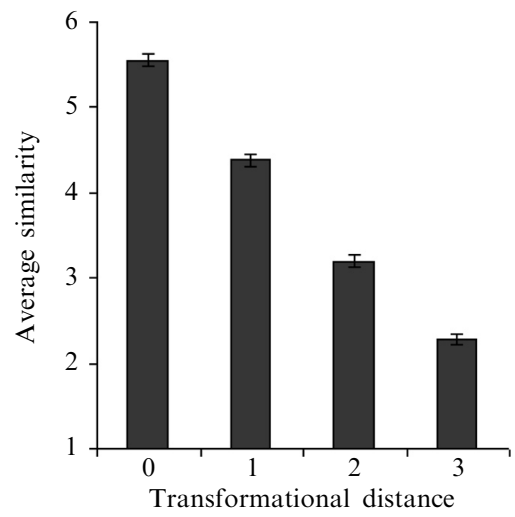

Figure 3. Rated similarity between two exemplars as a function of the transformational distance between them, averaged across categories, sequences, and trial types. In this and subsequent graphs the error bars represent the standard error of the mean. 
significant and negative Spearman correlation between transformational distance and the average similarity ratings $\left(r_{\mathrm{S}}=-0.965, p<0.0001, N=80\right)$. A very strong linear trend $\left(F_{1,57}=4224.11, p<0.0001\right)$ and a weaker but highly significant quadratic trend $\left(F_{1,57}=11.4, p<0.002\right)$. The cubic trend was not significant $\left(F_{1,57}=2.67\right.$, $p=0.1081)$. Pairwise multiple comparisons showed that all the means were significantly different from each other.

For each combination of subject and category, the Spearman rank correlation $(N=24)$ between average rated similarity (across trials with equal distance) and the transformational distance for each sequence $(0,1,2,3)$ was highly significant; the average Spearman rank correlation across these 220 combinations equaled -0.9 $(\mathrm{STD}=0.05)$. For each of the 66 morphing sequences, the Spearman rank correlation $(N=4)$ between average similarity (across subjects and trials) and transformational distance between stimuli equaled -1 .

Finally, the mean ratings of individual subjects (averaged across the 2 to 6 trials with the same distance) for each of the $(66 \times 4)$ morphing sequence-positions were examined. For the 1320 subject-specific morphing sequences ( 66 sequences $\times 20$ subjects), 128 morphing sequences $(9.7 \%)$ showed one or two deviations from strict monotonicity. When considering all the possible pairs $(N=3960 ; 20$ subjects $\times 66$ morphing sequences $\times 3$ pairs per morphing sequence), 134 pairs $(3.38 \%)$ deviated from strict monotonicity.

\subsection{Discussion}

The results of experiment 1 show that the topological transformations created by the morphing algorithm systematically influenced the judged similarity between stimuli, as found by Graf (2002) and predicted by TMRC and the structural alignment account of similarity (Hahn et al 2003; Markman and Gentner 1993a, 1993b). The similarity ratings decreased systematically with increasing transformational distance between the exemplars.

However, although Graf (2002) found a significant linear trend, as we did, he does not report the quadratic trend which is also significant in our data. The quadratic trend is also present visually in Graf's data. We believe that the significant quadratic trend and the observed deviations from monotonicity for individual subject data are more consistent with the conception of similarity as an exponentially decreasing function of distance.

Anyway, we can conclude that people usually could discriminate the shape changes and that the amount of change could also be translated into corresponding similarity ratings. Because the specific type and amount of transformation between exemplars was different for different morphing sequences (see figure 2), and because the monotonic relation between transformational distance and average rated similarity was found for every morphing sequence, this correspondence is far from trivial and seems to imply the involvement of considerable cognitive processing (see also Nosofsky 1986). For instance, participants seem to adjust the range of the rating scale to the range of the variability within a morphing sequence or category.

\section{Experiment 3. Sequential basic-level matching and topological transformations}

\subsection{Introduction}

Exemplar-based theories can and have been extended to account for categorisation performance. For example, Nosofsky and Palmeri (1997a, 1997b) introduced the exemplar-based random-walk model (EBRW) in which categorisation decisions are made by retrieving stored exemplars from memory. These sequentially retrieved exemplars drive a randomwalk process in which evidence builds up to one of the alternative responses over time. 
In Lamberts's (2000) model, an accumulation of perceptual information about the values of the input stimulus on different shape dimensions is used to predict categorisation performance, resulting in a similarity measure that is also time-dependent.

In experiment 3, we used a sequential matching paradigm to investigate the effect of transformational distance on sequential basic-level matching performance. According to TMRC, basic-level categorisation is achieved by a topological transformation process, which aligns the category representation and the stimulus representation. The transformation process is time-consuming, error-prone, and analogue (ie it traverses intermediate points in the transformational path). Consequently, TMRC predicts (a) a systematic (monotonic) relation between response latencies (and errors) and the extent of topological transformation (ie longer latencies and higher error rates for larger topological transformations), and (b) sequentially additive transformation times.

According to the particular exemplar-based model of Cohen and Nosofsky (2000), performance in sequential matching tasks (ie speeded same-different judgments) can be modeled by assuming that pairs of stimuli are memorised and retrieved to drive a random-walk process. One of the predictions of this model is that same responses are faster for objects lying in isolated than those lying in dense regions of similarity space.

\subsection{Methods}

4.2.1 Participants. Twenty-two university undergraduates in psychology participated voluntarily (mean age 22 years) and were tested individually in two sessions of around $50 \mathrm{~min}$ each.

4.2.2 Procedure. Each trial started with a fixation cross for $500 \mathrm{~ms}$, followed by the first stimulus (150 ms), a mask for $500 \mathrm{~ms}$, the second stimulus $(150 \mathrm{~ms})$, and a mask until response. Subjects had to decide whether both stimuli belonged to the same basiclevel category (same trial) or not (different trial). The experiment consisted of 1056 same trials and 1056 different trials. The former were created by pairing the exemplars from each sequence with each other $(4 \times 4 \times 6$ sequences $\times 11$ categories $=1056$ same trials $)$. The latter were created by pairing the first stimulus from all the same trials with a stimulus from another category. For each subject, the 2112 trials were presented randomly and divided into two sessions. Before the experiment, subjects were made familiar with the category labels, the kind of stimuli (ie outlines that were not extreme in the MDS solution) and the task (44 practice trials). Stimuli $(400 \times 300$ bitmaps $)$ were shown centred on a 15 -inch laptop screen $(1024 \times 768$ pixels, $60 \mathrm{~Hz}$ refresh, background luminance $20 \mathrm{~cd} \mathrm{~m}^{-2}$ ) and subtended $12.2 \mathrm{deg} \times 8.9 \mathrm{deg}$. A chin-rest was placed $57 \mathrm{~cm}$ away from the screen. Trials were self-paced. Reaction time and accuracy were recorded. No feedback was given. Subjects were instructed to respond as fast and accurately as possible.

\subsection{Results}

4.3.1 Reaction time. In 4.27\% $(N=993)$ of all same trials $(N=23232)$ an error was made, leaving 22239 correct same trials. Reaction times that were larger than $2500 \mathrm{~ms}$ and smaller than $200 \mathrm{~ms}$ were excluded (ie 52 trials or $0.23 \%$ of the correct same trials) leaving 22187 trials to enter the analyses. The grand mean RT equaled $511 \mathrm{~ms}$ and was comparable to the $471 \mathrm{~ms}$ found by Graf (2002). This increase of $40 \mathrm{~ms}$ may be due to the fact that our stimuli were on average more complex than those used by Graf (2002).

First, we calculated the average RT for each combination of subject, category, session, and transformational distance. These average RTs were analysed by a repeated-measures ANOVA with category ( 1 to 11$)$, session $(1,2)$, and transformational distance $(0,1,2$, and 3) as within-subject factors. Results showed a significant main effect of category 
$\left(F_{10,210}=8.55, p<0.0001\right)$, transformational distance $\left(F_{3,63}=67.83, p<0.0001\right)$, and session $\left(F_{1,21}=43.96, p<0.0001\right)$. RTs increased with increasing transformational distance and decreased from session 1 to session 2 (figure 4). Trend analysis for transformational distance showed a significant linear $\left(F_{1,63}=196.88, p<0.0001\right)$ and quadratic trend $\left(F_{1,63}=6.41, p<0.014\right)$, but no significant cubic trend $(F<1)$. Tukey - Kramer corrected pairwise multiple comparisons for the levels of distance showed significant differences between all pairs.

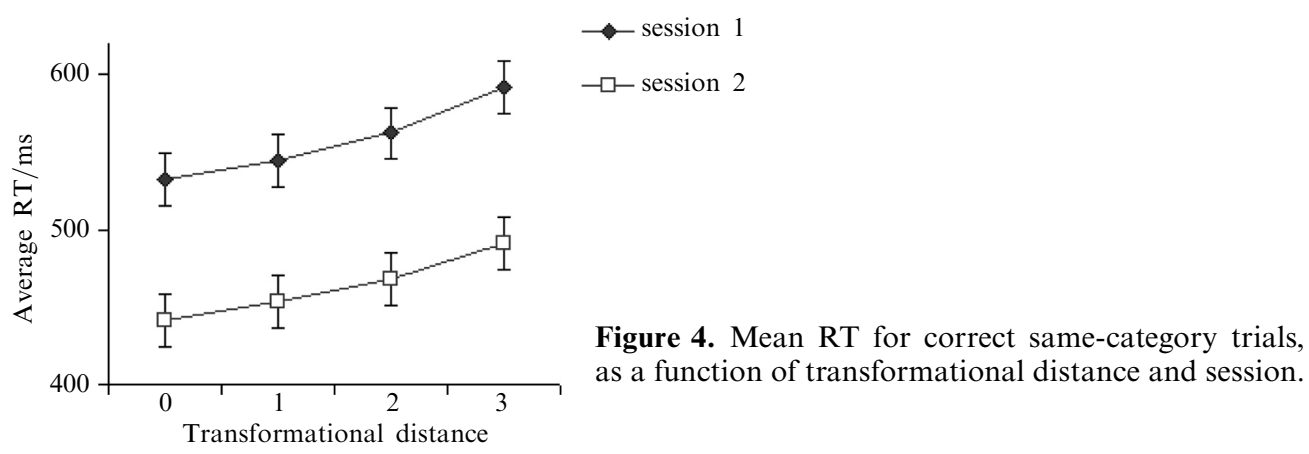

However, the interaction between distance and category was also significant $\left(F_{30,1533}=2.02, p<0.001\right)$. Therefore, we subsequently analysed the data separately for each category to test the effect of transformational distance for each of the six morphing sequences in each category. For all categories there was a significant overall effect of transformational distance. However, for 49 out of the 66 morphing sequences (74\%), the effect of transformational distance on RTs was not significant. Also, only 14 morphing sequences $(21 \%)$ showed a monotonic relation between transformational distance and RT.

In figure 2 (second column) we show the average RT for each stimulus for the same distance-0 trials. The maximum (white) and minimum (black) RT for each category is indicated, as well as local minima (grey; by comparing the three neighbours for each extreme exemplar and the two neighbours for each morphed exemplar). In contrast to the prediction of Cohen and Nosofsky (2000), the same response in distance- 0 trials to extreme exemplars lying in an isolated region (ie with rank 1 in figure 2, first column) was not systematically the fastest; for three categories (butterfly, bird, motorcycle) it was even the slowest. However, it is likely that the rank-1 exemplars are not necessarily isolated in the underlying psychological space employed by the subjects.

4.3.2 Error rates. For each subject, we calculated the percentage error for each transformational distance. A one-way ANOVA showed a significant effect of transformational distance on error rate for same-category trials $\left(F_{3,63}=18.4, p<0.0001\right.$; figure 5). The effect of session $\left(F_{1,21}=1.25, p=0.28\right)$ and the interaction $\left(F_{3,63}=1.1, p=0.36\right)$ were not significant.

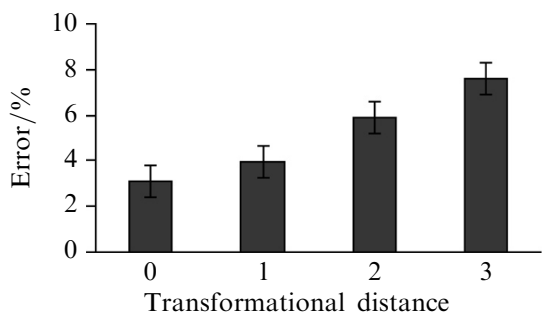

Figure 5. Error rate as a function of transformational distance for the same-category trials. Errors increase systematically when people have to respond "yes" to increasing shape differences. 
4.3.3 Sequential additivity. In order to investigate whether the RTs were sequentially additive, which would point to analogue transformations, we followed the procedure of Graf (2002), and first determined 'pure' transformation times for each trial type containing different exemplars. For example, for the analyses across subjects, we first calculated the average RT for each combination of subject and the 16 trial types (averaged across categories and sequences). Data for trial types with the same exemplars were collapsed, resulting in 10 trial types: ' 11 ', '22', '33', and '44' with distance-0, '12' (collapsed with 21), '23' (collapsed with 32), and '34' (collapsed with 43) for distance-1, '24' (collapsed with 42) and '13' (collapsed with 31) for distance-2, and finally '14' (collapsed with 41) for distance-3. Next, pure or 'observed' transformation times were calculated. For example, the observed transformation time for trial type 12 (TT12) equals the average RT for 12 (RT12) minus the average of RT11 and RT22. Similarly, TT13 equals RT13 minus the average of RT11 and RT33, and so on. Next, we calculated the predicted transformation times for each of three distances: the predicted TT13 $=$ TT12 + TT23, the predicted TT24 $=$ TT23 + TT34, and the predicted TT14 $=$ TT12 + TT23 + TT34. Scatter plots of the predicted and observed TTs for this analysis across subjects are shown in figure 6 (left column) for each of the three distances. Correlations between observed and predicted transformation times for distances 13, 24, and 14 equalled 0.75, 0.47, and 0.46, respectively (all $p \mathrm{~s}<0.05 ; N=22$ ). $t$-Tests showed that the difference in mean transformation times between predicted and observed times was almost significant for the largest distance (distance 14: $t_{21}=1.76$, $p=0.093$ ) but not for distances 13 and 24. A similar analysis was done for the 66 morphing sequences (figure 6, right column). Here, the correlations for 13, 24, and 14 equalled 0.59, 0.46, and 0.46, respectively (all $p \mathrm{~s}<0.05, N=66$ ). Again, the difference in mean transformation times between predicted and observed times was almost significant for the largest distance (distance 14: $t_{65}=1.76, p=0.082$ ) but not for distances 13 and 24. Note that several predicted transformation times were negative, indicating that the mean RT for distance-1 trials (ie RT12, RT23, and RT34) was lower than for distance-0 trials. Interestingly, also several observed transformation times were negative, but only for the analysis across morphing sequences (figure 6, right column).

\subsection{Discussion}

In experiment 3 , error percentages and average reaction times increased monotonically with increasing transformational distance between both exemplars in a sequential basiclevel matching task, as found by Graf (2002).

However, in our data set, the hypothesis of sequential additivity, which lies at the heart of TMRC, was violated many times, since (i) for $74 \%$ of the sequences no significant differences in RTs were found; (ii) only $21 \%$ of the morphing sequences showed a monotonic relation between transformational distance and RT; (iii) the correlations between predicted and observed transformation times were not very high; and (iv) for many sequences the predicted and/or 'observed' transformation times were negative. The evidence for sequential additivity was also not very strong in Graf's data (which he himself also admitted-Graf 2002, pages $142-143$ ).

To account for sequential matching performance (ie speeded same-different judgments), Cohen and Nosofsky (2000) extended the EBRW of Nosofsky and Palmeri (1997a) by assuming that two processes drive the same-different judgments. First, whenever a pair of objects is presented, subjects are assumed to make on-line judgments of perceptual similarity; high values drive a random-walk counter toward a 'same' response criterion, while low values tend to drive the counter toward a 'different' response criterion. Second, observers are also assumed to store individual examples of experienced same and different pairs of objects in memory. When presented with an input pair, exemplar pairs from memory are retrieved according to their similarity to the input pair; 

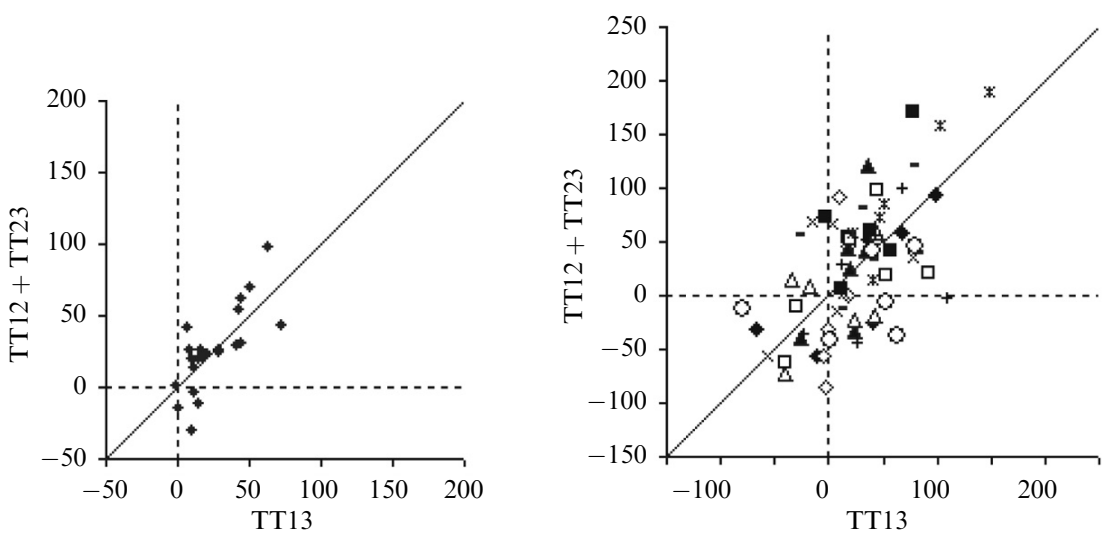

+ car
$\square$ chair
$\varnothing$ bird
$\diamond$ guitar
+ vase
$\star$ beetle
$\times$ fish
$\Delta$ rodent
$*$ airplane
amotorcycle
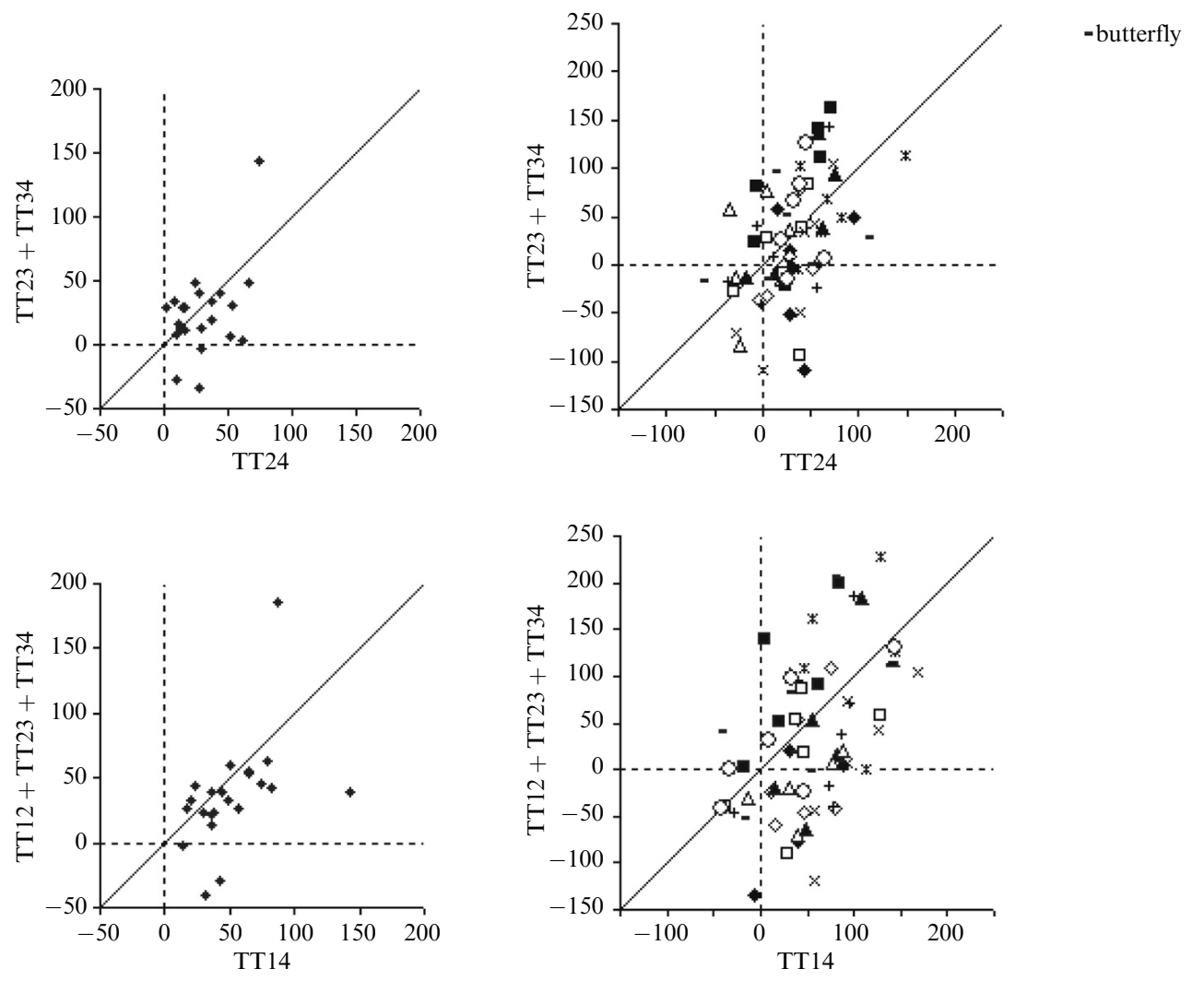

Observed RT/ms

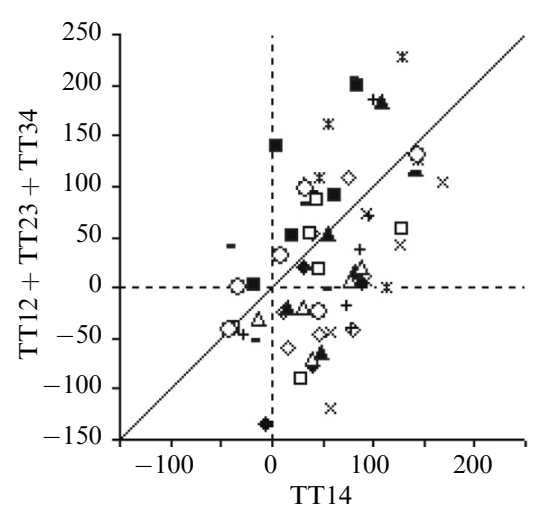

Observed RT/ms

Figure 6. (left) Scatter plots of the predicted and observed transformation times (TT) of twentytwo subjects for each of three distances $(13,24$, and 14). (right) Scatter plots of the predicted and observed categorisation times of 66 morphing sequences for each of three distances (13, 24, and 14; both directions). Different symbols indicate different categories.

retrieval of same (different) pairs moves the random walk toward the same (different) response criterion.

Thus, as the transformational distance between two outlines increases, the initial on-line judgment of perceptual similarity will tend to drive the counter toward a 'different' response criterion. However, since similarity is time-dependent (Lamberts 2000), continued visual processing will eventually drive the counter toward the 'same' response criterion, and this will take longer when the transformational distance is larger, producing 
the monotonic increase in RT with increasing transformational distance. The improvement in RT from the first to the second session is then due to the fact that the visual stimuli are stored in memory (during session 1) and come to speed up the decision process (during session 2). In other words, the sequential matching task becomes more automatic when the experience of the observers with individual exemplars and pairs of exemplars increases (see also Palmeri et al 2004).

Although this explanation sounds valid, we believe that it does not accurately describe human performance in sequential matching tasks. TMRC, EBRW, and Lamberts's (2000) information-accumulation model have one thing in common: responses are only initiated after a decision process. In section 6 we will discuss an alternative explanation according to which the effect of image transformations on sequential matching performance measures is localised, at least in part, at the response level.

\section{Experiment 4. Typicality and topological transformations}

\subsection{Introduction}

In experiment 4, we collected typicality ratings for each of the 16 exemplars of each category to assess the ecological validity of our morph stimuli in each category. If the morph exemplars are interpreted as real exemplars from a category, their typicality should be at least as high as that for the selected extreme exemplars.

Furthermore, TMRC and exemplar-based models generate contrasting predictions. According to TMRC, typicality is based on the transformational distance to the category prototype representation. The most typical stimulus is regarded as being the most similar to the category prototype representation. TMRC predicts that typicality ratings will decrease with increasing distance from the most typical exemplar (the prototypical exemplar) in a morphing sequence. Since categorisation of a stimulus is based also on the topological distance, TMRC predicts an association between typicality and categorisation (ie higher typicality is associated with faster RTs and vice versa).

In contrast, exemplar-based models assume that typicality is based on the summed similarities of the stimulus to all the exemplars from that category, while categorisation is determined by the relative degree of target-category in contrast-category similarity. Therefore, a dissociation between classification and typicality judgments can be expected, as has been observed by Nosofsky (1988).

Interestingly, it is generally assumed that category prototypes constructed to be physical central tendencies of category exemplars are also psychological central tendencies (eg Smith and Minda 2001). While this assumption of a fairly direct mapping between physical and psychological dimensions may exist for stimuli with clearly defined psychological dimensions (eg semicircles of varying sizes containing radial lines of varying angles), Palmeri and Nosofsky (2001) argued that the mappings between physical properties and psychological dimensions are not so clearly defined for fairly complex stimuli (eg artificial dot-patterns, checkerboard patterns, and perhaps also for more natural stimuli). The result is that prototypes that are physical central tendencies of category exemplars may sometimes reside not as psychological central tendencies, but as psychological extreme points relative to the category exemplars (and to the exemplars of contrast categories). In each of three experiments, Palmeri and Nosofsky (2001) created prototypes (dot patterns in the shape of a triangle, a plus, and an F; three unfamiliar dot patterns with vertical symmetry; two complex checkerboard patterns) and distortions of these prototype patterns, the latter acting as category exemplars. During training in each experiment, participants learned to classify a subset of the distortions with feedback. During transfer, participants were tested on the old distortions, on new distortions, and on the prototypes. Finally, in each experiment, participants made pairwise similarity judgments between all possible pattern 
pairs to derive the psychological coordinates of all the patterns using MDS. The most important result in the current context is that the MDS solutions of each experiment showed that the prototypes were psychological extremes in relation to other exemplars of their categories (and to the exemplars of contrast categories). During categorisation learning with complex physical stimuli, various emergent dimensions, based on diagnostic, functional features may be formed, which causes the prototypes to be represented as psychological extremes. The similarity measure based on these features can be quite different from the similarity measure used to create the physical central tendencies. The MDS solutions also allowed exemplar-based models to predict the observed extreme prototype-enhancement effects better than prototype-based models.

Based on this reasoning, it is possible that participants have created such functional features during categorisation learning of real-life objects, and that the exemplar outline rated as most typical will occupy extreme positions.

\subsection{Methods}

5.2.1 Participants. Fifty-six high-school students participated voluntarily (mean age 17 years) and were tested collectively in one of three groups. The experiment lasted around $30 \mathrm{~min}$.

5.2.2 Procedure. The 16 exemplar outlines (4 extremes +12 morphs) of each category were printed on one A4 page, in a random order for each participant. For each of the eleven categories, subjects had to rate the typicality of each exemplar on a 6-point scale (1 very atypical 6 very typical) which was also printed on each page. Subjects were encouraged to use all points of the scale.

\subsection{Results}

First, we calculated the average typicality for each combination of subject and the four exemplar positions within a sequence. Like Graf (2002), we did not necessarily expect that the effect of position would be significant because the position of the category prototypes might be distributed over categories and sequences. Still, a oneway repeated-measures ANOVA showed a significant effect of exemplar position $\left(F_{3,159}=18.576, p<0.0001\right)$. On average, the typicality ratings were higher for exemplars in the middle positions than for the exemplars at the extreme positions of the sequences (figure 7). Trend analyses showed a significant linear trend $\left(F_{1,159}=16.69\right.$, $p<0.0001)$ and an even stronger quadratic trend $\left(F_{1,159}=39.6, p<0.0001\right)$, but no significant cubic trend $(F<1)$. Pairwise multiple comparisons showed that positions

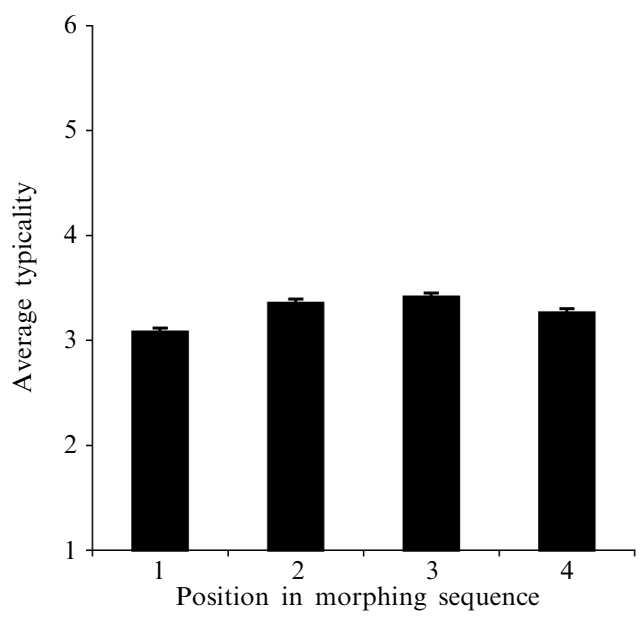

Figure 7. Rated typicality for exemplars at each position in a sequence, averaged across categories, morphing sequences, and trial types. Extreme exemplars occupy the first and fourth positions; morphed exemplars occupy the second and third positions. Note that the average typicality for positions 1 and 4 differs because only one direction of positions in a morphing sequence was used (ie $1-2-3-4$, or $4-3-2-1)$. 
2 and 3, and positions 2 and 4 did not differ significantly from each other, while the others did.

Second, to investigate the prototypicality gradients for each sequence in each category, figure 2 (right column) shows the mean typicality ratings of each exemplar of each category (averaged across subjects) with the maximum (black) and minimum (white) values indicated. Similar to Graf (2002), we define a prototypical exemplar as an exemplar for which the average typicality is higher than that of their neighbouring exemplars (ie three neighbours for each extreme exemplar and two neighbours for each morphed exemplar; grey squares in figure 2 right column; the black square indicates maximum typicality). Based on this definition, the number of prototypical exemplars for a category ranged from 1 to 6 . Figure 8 shows the position of the four extremes for each category when the sixteen stimuli are sorted from low to high typicality.

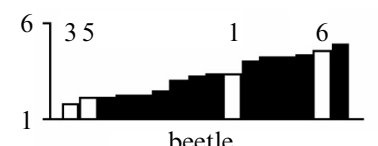

beetle
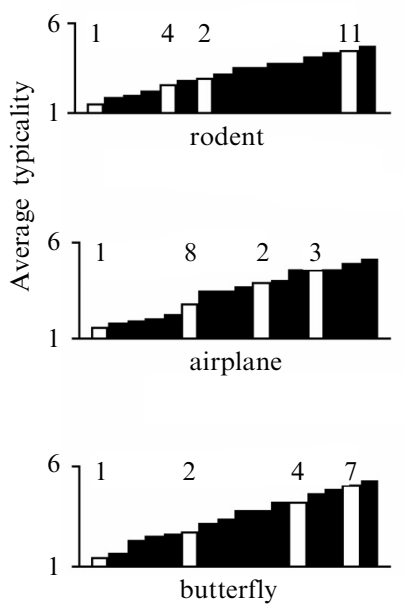
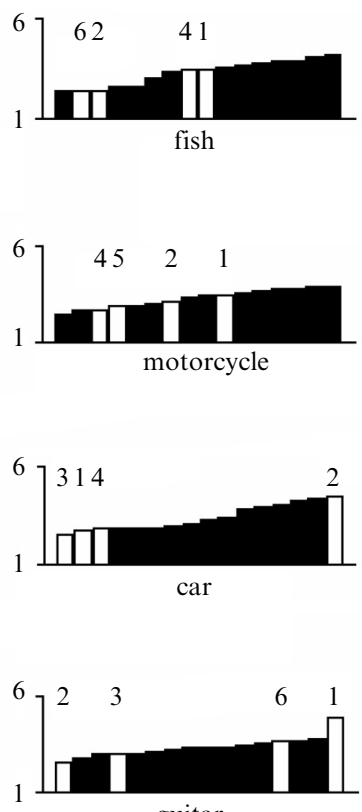

guitar

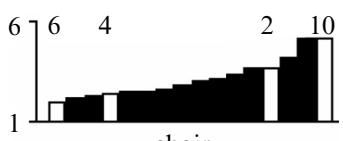

chair
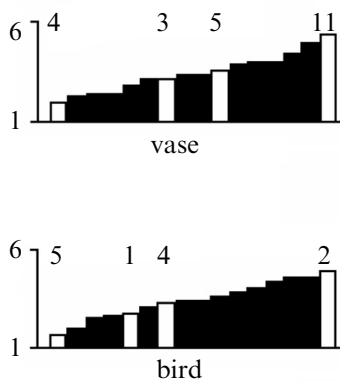

Figure 8. Positions of the four extreme exemplars (white) and the morphed exemplars (black) when stimuli are sorted from low to high typicality. Numbers on top correspond to the rank of the extreme exemplars in figure 2.

Two interesting observations can be made. First, a clear dissociation between typicality and categorisation is evident when comparing the positions of the white, black, and grey squares in the middle and right column of figure 2. TMRC would predict the same locations of the different coloured squares in both columns for each category. Second, figure 8 shows that the exemplar that was rated as the most typical occupied an extreme position for five out of eleven categories, while this was also the case for the second-most typical for three other categories. For nine categories the exemplar rated on average as least typical was also an extreme stimulus.

\subsection{Discussion}

In experiment 4 we found that the rated typicality was on average higher for the intermediate morph stimuli than for the extreme stimuli, replicating the findings of Graf (2002). The position of the exemplar that was rated as most typical, however, was an extreme position for five out of eleven categories (car, guitar, chair, vase, bird). 
This finding is inconsistent with the idea that the category prototype representation is the central tendency of the exemplars, and therefore should occupy a central position in the underlying psychological space. However, just as exemplar-based models, TMRC allows the existence of multiple prototypes (in analogy with multiple views in object recognition). The observed range of (local) prototypes across categories (one to six) might resemble the existence of a different number of subcategories for different basic-level categories. Crucially, the observed dissociation between typicality and categorisation clearly favours an exemplar-based account over an alignment account.

Finally, the observation that an extreme exemplar was rated as most typical for five categories is not inconsistent with the idea put forward by Palmeri and Nosofsky (2001) who, as mentioned in section 1, found that prototypes that are physical central tendencies of category instances may become represented psychologically as extreme points. They suggested that the extreme-point prototype representation might arise from the emergence of diagnostic, functional features that may be created as part of the process of category learning (Schyns 1997, 1998; see also Schyns and Rodet 1997). Neurophysiological studies showed that neurons in IT respond strongest to stimuli with extreme values of shape dimensions (De Baene et al 2007, 2008; Kayaert et al 2005). Thus, although prototypes are never observed during natural vision, the prototypes used in vision research (eg stimuli with perfect symmetry, averages of a large number of faces, etc), and our exemplars that are rated as most typical, might have extreme values of the diagnostic shape dimensions from exemplar-based models.

\section{General discussion}

We created and validated a new stimulus set using morphing between four selected exemplar outlines from the same category, and investigated the predictions from a transformational alignment model and from exemplar-based models. The nonlinear relation between similarity and topological distance (experiment 2), the deviations from sequential additivity (experiment 3), and the dissociation between typicality and categorisation (experiment 4), contradict the idea of an analogue transformational alignment process, but not of exemplar-based representations.

However, we believe that current exemplar-based categorisation models do not yet adequately describe human visual performance. In this section we first discuss two principles - the world as an outside memory and the continuous-flow model of information processing - that have been neglected in the literature. Subsequently, we argue that when exemplar-based models also incorporate the second principle, they can quite easily explain the behavioural dependency on all kinds of image transformations. Next, we discuss the concept of transformation and its relation to gain-modulation. We end by discussing other time-consuming and error-prone processes that can contribute to the behavioural dependence on image transformations.

\subsection{The world as an outside memory}

O'Regan (1992) noted, as many before him, that, despite all kinds of defects in the construction of the eye and retina (eg the fovea is not a region of uniform acuity), the world we perceive does not seem of different resolution or colour at different eccentricities, and the displacement of the retinal image caused by saccades and fixational instability is usually not noticed. Traditional theories, including TMRC, incorporate some kind of compensatory mechanisms that implicitly assume the existence of an internal representation like a kind of 'internal screen' or 'scale model' which has metric properties like the outside world. However, O'Regan (1992) discusses experiments that suggest an alternative view in which the outside world is considered a form of ever-present external memory that can be sampled at leisure through eye movements. According to this view there is no need to reconstruct shape 
to compensate for eye movements or image transformations, and not many details are remembered across saccades. The goal is simply to extract enough visual information to decide on an appropriate action. Vision is an active process where eye movements cause a change in sensation which is used to modify or confirm an interpretation, which guides further action (O'Regan 1992; see also Rybak et al 1998; Trick and Enns 1997).

While TMRC and classical structural description theories such as recognition-bycomponents (Biederman 1987) reconstruct shape or parts, and thus represent shape information through the similarity between input and memory (first-order isomorphism), exemplar-based models do not try to reconstruct shape but convey information about shape by representing the similarity between shapes itself (second-order isomorphism Edelman 1998) by means of an interpolation process (see above). Nevertheless, the structure of objects can be represented in exemplar-based models if shape-tuned units are also selective to the location of its preferred shape feature ('what + where' unitsEdelman and Intrator 2000; see also Newell et al 2005). In this view, exemplars are defined as image fragments (in contrast to holistic images or generic parts), and the spatial relations between them are continuous and coarsely coded. In general, features that co-occur more frequently will become more strongly associated, and extensive experience with the same features in a consistent configuration will give rise to more complex features. In contrast to rigid templates, the relative positions of features might be represented probabilistically instead of deterministically, thereby providing flexible or 'deformable' templates for recognition (Barenholtz and Tarr 2008; Tarr and Bülthoff 1998).

For example, Kukkonen et al (1996; see also Wagemans et al 1996, 2000) showed that relatively simple cues, such as parallelism, collinearity, and curvature sign (convexities versus concavities), which are easy to extract during different fixations, are used when judging whether two retinal images arise from the same object viewed from different positions. These image-based properties, resulting from perceptual organisation, can be described as 'qualitative or quasi-invariant properties' - by themselves special cases of mathematical invariant properties - and might partly underlie the fast assessment of affine, projective, and Euclidean shape equivalence, and topological class equivalence.

\subsection{The continuous-flow model of human information processing}

The continuous-flow model of information processing (Eriksen and Schultz 1979; see also Coles et al 1985) suggests that the latency and accuracy of overt behavioural responses are a function of (a) a response activation process controlled by a stimulus evaluation process that accumulates evidence gradually, (b) a response priming process that is independent of stimulus evaluation, and (c) a response competition process. Although this model was invented in the context of visual search tasks, where RT increased with increasing number of distractors, we will elaborate on these processes and provide recent studies that are consistent with the predictions of this model, although they were not set up to test them directly. Current exemplar-based categorisation models are largely neglecting these processes.

First, Eriksen and Schultz (1979) discuss how improvement in acuity with increased viewing time or exposure duration of the stimulus can be attributed in most instances to increased energy summation over the longer temporal interval. Such summation of energy implies that the accumulation of information about a stimulus in a sensory register or the percept is a temporally distributed process. The gradual accumulation of stimulus information in the visual system is consistent with (a) the gradual increase in neural activity of single cells and cell populations in object-related areas (Eger et al 2006; Mruczek and Sheinberg 2007), (b) the reverse hierarchy theory (Hochstein and 
Ahissar 2002) which states that the local properties or details are only available later when focused attention is directed to them, (c) the finding that object-selective neurons first convey global, categorical information before they convey local, fine identity information (Sugase et al 1999), and (d) recent computational and neurophysiological research that uses time as a coding dimension for neural representations (Perrett et al 1998; Wyss et al 2003). For example, Perrett et al suggest that the speed of recognition of an object depends on the rate of accumulation of activity from responsive neurons that is evoked by the input view, which depends on the extent that the object has been experienced before under the same viewing conditions. Activity among the population of cells selective to the object's appearance will accumulate more slowly when the object is seen in an unusual view, orientation, or size, compared to the training experience. Furthermore, if the evidence for a particular object at a particular orientation is small (because of degraded input or short presentation durations) more information needs to be gathered by covert and overt attentional selection processes (Perrett et al 1998). However, it is not clear whether such a process is sufficient to explain the behavioural dependence on image transformations in sequential matching tasks.

Second, according to the continuous-flow model, response activation begins as soon as some visual information is accumulated. Thus, the process of stimulus evaluation continuously feeds information about the stimulus to associated response activation systems. Early in the process, the information is consistent with a wide range of responses, and these receive initial activation. As the information continues to accumulate, response activation becomes increasingly focused on responses that remain viable alternatives, given the accumulated data. A given response is only evoked when the activation of its channel exceeds a threshold. Crucially, responses can compete with each other. The speed with which a correct response is executed depends, in part, on the extent of response competition. The greater this competition, the longer the latency of the correct response.

Thus, this continuous-flow model does not provide for a separate decision stage responsible for activating or initiating responses; responses are emitted whenever one of the response channels exceeds a criterion level (which can fluctuate from trial to trial). Furthermore, it implies that the duration of the evaluation process cannot always be inferred from RT (which is an implicit assumption in TMRC). Initial evidence for these processes in a non-search task comes from a study of Coles et al (1985) who used an analogue-response device (two dynamometers) and measures of the electromyogram of the hand muscles to show the reality of partial response activation and response competition. By measuring also the latency of the P300 component of the eventrelated brain potential (probably related to a process that is invoked only after stimulus evaluation has been completed), Coles et al (1985) could confirm the predictions of the continuous-flow model.

Recent evidence for response competition in the context of a categorisation task comes from Spivey and Dale (2006) and Dale et al (2007) who used eye-tracking and mouse-tracking evidence. Also, neurophysiological and neuroimaging research on decision-making is consistent with the idea of a continuous flow of information up to response systems (Heekeren et al 2004; Hernández et al 2002; McKeeff and Tong 2007; Rorie and Newsome 2005). For example, Hernández et al (2002) trained monkeys to discriminate between two vibrations applied sequentially to the fingertips; monkeys had to recall the frequency of the first vibration, compare it to the second one, and indicate with a hand movement which of the two vibrations had the higher frequency. They found that the responses of single neurons in medial premotor cortex correlated with these diverse stages of the discrimination process (ie coding the first, coding the second, comparison, response). Finally, the idea that multiple object representations can be activated and compete has recently been taken up by Bar (2003), 
Bar et al (2006), Gabroi and Lisman (2003), Panis et al (2008a), and Panis and Wagemans (2008). For example, Gabroi and Lisman (2003) show how bidirectional flow of information in reciprocally connected hierarchical cortical areas can be organised to produce recognition of objects and categories through the detection of combinations of features, and how the serial process of attention can be integrated with the parallel recognition processes. After the early activation of a set of candidate objects (and responses) based on early and coarse information (eg low spatial frequencies), later bottom - up flow of detailed information through a narrow window of attention then leads to the inactivation (exclusion) of candidate object representations (eg activated flexible hierarchical feature templates) that are inconsistent with the sampled information, thereby reducing the set of possible objects and responses. Algorithms for moving attention make use of top-down connections to compute the relative probability of each feature or spatial relation between features, given the set of still-possible object representations, after which attention will move to the most informative feature. Recognition occurs after a few cycles when the serially sampled information leads to the inactivation of all but one candidate object (Gabroi and Lisman 2003).

Third, another way in which response channels can be activated is through a response-priming process that is independent of the nature of the input stimulus and may even precede stimulus presentation. Such aspecific priming may be triggered by factors such as instructions, expectancy, pay-off schedules, and the like (Coles et al 1985).

We argue that the continuous-flow model can, at least in part, explain the behavioural dependence on topological and other image transformations. In particular, the increase in RT (and error percentage) with increasing topological distance in experiment 3 is caused by a greater competition between the responses of both hands: 'yes' (same) or 'no' (different). Response competition is lowest in distance-0 trials since there is no evidence in the stimuli for the 'no' response. However, a low number of errors is made because of the aspecific priming process, which will sometimes activate the incorrect response. For distance-1 to distance-3 trials, the initial evidence is increasingly activating the 'no' response because of the greater shape changes and the image-based sensitivity of the visual system, leading to stronger response competition, and the observed higher error probabilities and latencies of the correct 'yes' response.

Furthermore, negative transformation times for distance-1 trials (TT12, TT23, or TT34), resulting in negative subject- and sequence-specific, predicted transformation times for distance-2 and distance-3 trials (TT13, TT24, and TT14), are not surprising: trial-by-trial fluctuations in the threshold and/or fluctuations in the amount of aspecific priming can result in a faster crossing of the threshold in some distance-1 trials compared to distance- 0 trials, when the competition is weak. However, when the shape changes reach a critical size (as in distance- 2 and distance- 3 trials), the competition between responses is strong enough to prevent a quick crossing of the threshold of the correct response; no observed subject-specific transformation times (averaged across all morphing sequences) for distance- 2 and distance-3 were negative (figure 6 left column). In contrast, observed morphing sequence-specific transformation times for distance- 2 and distance- 3 trials can be negative because they are averaged across subjects which differ in the speed of information transmission, the threshold level, the amount of aspecific priming, category knowledge, muscle strength, etc.

\subsection{Transformations and gain-modulation}

Although our data reject TMRC, analogue transformations of visual information might occur in the system. Information processing of visual information can be considered as a progressive series of visual re-representations or transformations of the retinal representations, from V1 to V2 to V4 to IT, in order to make objectively similar stimuli closer to each other, and dissimilar stimuli farther apart (Connor et al 2007; 
DiCarlo and Cox 2007; Weiss and Edelman 1995). Furthermore, gain-modulation is a nonlinear way in which neurons combine information from two or more sources, and it can serve as a basis for a general class of computations, namely coordinate transformations and the generation of invariant responses (Salinas and Sejnowski 2001). For example, the receptive fields of some neurons in parietal cortex are gain-modulated by head and eye positions, resulting in a change from retinal, or eye-centred, to bodycentred coordinates. Also, neurons in V4 which respond to combinations of curvature and orientation, are gain-modulated by the location where attention is directed, and leads downstream IT neurons that are driven by the gain-modulated ones, to become responding in a coordinate frame centred on the location where attention is directed, ie they are insensitive to the absolute location of the input pattern or translation invariant (Salinas and Sejnowski 2001).

These attention-centred responses in V4 might explain the orientation-congruency effects as observed by Graf et al (2005). They presented a first stimulus for $104 \mathrm{~ms}$ immediately followed by a second stimulus for $82 \mathrm{~ms}$ (on average across subjects). Both stimuli were either in congruent or incongruent orientations. Six different objects could appear as the first target and eighteen objects as the second target. Furthermore, left-facing objects were flipped so that all objects were oriented to the right. The orientation-congruency effect found by Graf et al, namely higher naming accuracy of both objects in the correct order for congruent than for incongruent orientations, was independent of superordinate category membership and was found for objects with different main axes of elongation (eg tree versus fish).

Gauthier and Tarr (1997) and Tarr and Gauthier (1998) found (picture-plane and 3-D) orientation priming only for visually similar shapes. Indeed, in an exemplar-based system in which objects are represented in a viewpoint-dependent manner, including information of the shape and orientation or pose, the same view of two different exemplars of the same class may be more similar than two different views of the same exemplar. The most salient difference between the study of Gauthier and Tarr (1997) and that of Graf et al (2005) is the difference in stimulus duration. Presenting stimuli for as short a time as Graf et al (2005) did will not allow extraction of details of shape and location (ie full accumulation of visual information about shape and location). Rather than concluding that orientation congruency effects argue for models in which recognition is achieved by an adjustment of a perceptual coordinate system, we believe that a much more basic process such as gain-modulation by attention can be responsible. First, we assume that accuracy is mostly dependent on the correct recognition of the second object. Second, it is known that covert spatial attentionalselection effects start around $80 \mathrm{~ms}$ after stimulus onset, peaking about $20 \mathrm{~ms}$ later (Hillyard et al 1998). Third, if the first object, eg a fish rotated by $50^{\circ}$ in the picture plane, is categorised around $150 \mathrm{~ms}$ after its onset, the system knows that the feature on the upper right of the fixation cross (where covert attention might be focused at that moment) is the top of the fish (eg its dorsal fin or a sharp convexity pointing to the upper right). Thus, when suddenly the second object (eg a tree rotated by $50^{\circ}$ ) replaces the first object at $104 \mathrm{~ms}$ after the onset of the first stimulus, the top-down gain-modulatory influences based on the location of attention might still continue when initial and coarse shape and location information of (fragments of) the second stimulus reaches V4; this will lead to the correct interpretation of the location of the new input only when both objects have the same orientation regardless of superordinate or main-axis congruency (ie if this new feature on the upper right of the fixation cross is also located on top, then it must be a tree). Indeed, because only eighteen objects could appear as the second target (and each object was seen many times), accumulating details about the shape of the new feature-together with the interpretation of its coarse location-will often allow rejection of all the incorrect responses. 
In contrast, when the second stimulus has a different picture-plane orientation (eg a tree rotated by $140^{\circ}$ ), then interpreting the new feature as lying on top of the object will interfere with the recognition process.

\subsection{Other time-consuming and error-prone processes}

Response competition is not the only time-consuming and error-prone process that might lead to the observed behavioural dependence on image transformations. First, the statistical properties of images of a certain category (eg face, dog, car, etc) might allow easy or difficult generalisation performance (Jäkel et al 2007). For example, Vanucci and Viggiano (2000) examined the effects of plane rotations on the identification of line drawings of exemplars from three categories (animals, vegetables, and inanimate objects). While a clear effect of rotation on response time was found for animals (which have been seen usually in the upright orientation), no effect at all was found for vegetables (which have been seen in all orientations before) and only partial effects for inanimate objects. Interestingly, the minimum amount of visual information necessary to identify rotated stimuli also varied as a function of category.

Second, departures from a monotonic increase in RTs and/or error rates with increasing spatial transformations have been observed, resulting from practice with certain views of the same object, or of visually similar objects (Lawson and Humphreys 1998; Tarr and Gauthier 1998).

Third, previous experiences with objects will lead to repetition priming effects (Schacter 1992). This unconscious or implicit form of memory refers to the fact that object recognition is improved for repeated compared to new stimuli. Furthermore, repetition priming is shape-specific, gradual, and long-lasting; and reduced but not eliminated with changes in an object's exemplar (Vuilleumier et al 2002). Since Wiggs and Martin (1998) concluded that all changes in physical attributes that are essential to the representation of object form do influence perceptual priming, we conclude that this process could contribute to differences in RT in sequential matching tasks. Note that neurophysiological manifestation of repetition priming and familiarity can also explain asymmetries in similarity judgments (Op de Beeck et al 2003).

The result is that different processes might be used for different transformations. For example, the results of Lawson et al (2000) suggest that compensation for plane rotation starts relatively early in processing, before compensation for depth rotation has begun. First, plane-rotated stimuli may be processed differently from upright stimuli because spatial relations between features may be harder to extract for planedisoriented views of familiar objects, whereas local features may be analysed equally efficiently at all plane rotations. Also, certain perceptual attributes that can be extracted before the identification of an object (eg based on low spatial frequencies-Bar 2003) could provide important information about the probable orientation of the object in the plane, for instance, the orientation of any axes of symmetry, the main axis of extension, and the likely base of the object. In contrast, these attributes may not be as informative about the depth orientation of an object. Identifying objects rotated in depth may depend on additional perceptual processing (if a more accurate representation is required compared to canonical views) or prolonged memory retrieval (if, for example, only fragments of foreshortened views could initially be identified, since the bounding contour or outline is not diagnostic_Lawson et al 2000). It is also quite likely that the shape of the parts of foreshortened objects and the spatial relationships between them differ so radically from the correctly stored, view-specific object representations, that it takes longer to accumulate enough evidence to rule out all the activated but incorrect candidates (see also Panis et al 2008a; Panis and Wagemans 2008). Similarly, the finding that RTs to decide if two sequentially presented shapes are identical except for changes in picture-plane orientation or size are sequentially additive 
(Bundesen et al 1981) is also consistent with the idea that two sequential and dissociable processes are involved, eg attentional selection of a spatial area of a certain size (see also Cave and Kosslyn 1989) and the extraction of low spatial frequencies that contain information about the probable orientation (see above).

\section{Conclusion}

It is perhaps not surprising that view-based and exemplar-based theories do a better job in explaining categorisation data, since they store more information and therefore can convey more information about the stimulus during a given period in comparison to prototype-based models. In contrast to the idea that categorisation involves analogue frame coordinate transformations implemented by gain modulation, we conclude that our results and other findings are consistent with exemplar-based, view-specific accounts of recognition for matching the input with stored representations when taking into account well-established processes such as accumulation of visual information, perceptual organisation, attentional selection, covert model selection, perceptual priming, response priming, and response competition.

Acknowledgments. This research was supported by a research grant from the Fund for Scientific Research (FWO Flanders, G.0281.06). This study is also part of larger research programs with financial support from the University Research Council (GOA/2005/03-TBA and IDO/02/004). We want to thank Wouter De Baene, Céline Gillebert, Bart Ons, Hans Op de Beeck, Gert Storms, Wolf Vanpaemel, and Rufin Vogels, for interesting discussions regarding this study. We also want to thank one anonymous reviewer and Rebecca Lawson for interesting suggestions regarding a previous version of this manuscript.

\section{References}

Bar M, 2003 "A cortical mechanism for triggering top-down facilitation in visual object recognition" Journal of Cognitive Neuroscience $15600-609$

Bar M, Kassam K S, Ghuman A S, Boshyan J, Schmid A M, Dale A M, Hämäläinen M S, Marinkovic K, Schacter D L, Rosen B R, Halgren E, 2006 "Top-down facilitation of visual recognition" Proceedings of the National Academy of Sciences of the USA 103449 -454

Barenholtz E, Tarr M J, 2008 "Visual judgment of similarity across shape transformations: Evidence for a computational model of articulated objects" Acta Psychologica 128331 - 338

Biederman I, 1987 "Recognition-by-components: A theory of human image understanding" Psychological Review 94115 - 147

Bundesen C, Larsen A, Farrell J E, 1981 "Mental transformations of size and orientation", in Attention and Performance Eds J Long, A Baddeley (Hillsdale, NJ: Lawrence Erlbaum Associates) pp $279-294$

Bundesen C, Larsen A, Farrell J E, 1983 "Visual apparent movement: Transformations of size and orientation" Perception $12549-558$

Cave K R, Kosslyn S M, 1989 "Varieties of size-specific visual selection" Journal of Experimental Psychology: General 118 148-164

Chen L, 1985 "Topological structure in the perception of apparent motion" Perception 14 197-208

Chen L, 2005 "The topological approach to perceptual organization" Visual Cognition 12553 - 637

Cohen A L, Nosofsky R M, 2000 "An exemplar-retrieval model of speeded same-different judgments" Journal of Experimental Psychology: Human Perception and Performance 261549 - 1569

Coles M G H, Grattton G, Bashore T R, Eriksen C W, Donchin E, 1985 "A psychophysiological investigation of the continuous flow model of human information processing" Journal of Experimental Psychology: Human Perception and Performance $11529-553$

Connor C E, Brincat S L, Pasupathy A, 2007 "Transformation of shape information in the ventral visual pathway" Current Opinion in Neurobiology $17140-147$

Dale R, Kehoe C, Spivey M J, 2007 "Graded motor responses in the time course of categorizing exemplars" Memory \& Cognition 35 15-28

D'Arcy Thompson W, 1917/1942 On Growth and Form 2nd edition (Cambridge: Cambridge University Press; original edition, 1917)

De Baene W, Ons B, Wagemans J, Vogels R, 2008 "Effects of category learning on the stimulus selectivity of macaque inferior temporal neurons" Learning \& Memory $15717-727$ 
De Baene W, Premereur E, Vogels R, 2007 "Properties of shape tuning of macaque inferior temporal neurons examined using Rapid Serial Visual Presentation" Journal of Neurophysiology 97 $2900-2916$

Demeyer M, Zaenen P, Wagemans J, 2007 "Low-level correlations between object properties and viewpoint can cause viewpoint-dependent object recognition" Spatial Vision 20 79-106

De Winter J, Wagemans J, 2004 "Contour-based object identification and segmentation: Stimuli, norms and data, and software tools" Behavior Research Methods, Instruments, \& Computers 36 $604-624$

DiCarlo J J, Cox D D, 2007 “Untangling invariant object recognition" Trends in Cognitive Sciences $11333-341$

Edelman S, 1998 "Representation is representation of similarities" Behavioral and Brain Sciences $21449-498$

Edelman S, 1999 Representation and Recognition in Vision (Cambridge, MA: MIT Press)

Edelman S, Intrator N, 2000 "(Coarse coding of shape fragments) + (Retinotopy) $\approx$ Representation of structure" Spatial Vision $13255-264$

Eger H, Henson R N, Driver J, Dolan R J, 2006 "Mechanisms of top-down facilitation in perception of visual objects studied by fMRI" Cerebral Cortex 172123 - 2133

Eriksen C W, Schultz D W, 1979 "Information processing in visual search: A continuous flow conception and experimental results" Perception \& Psychophysics 25 249-263

Gabroi D, Lisman J, 2003 "Recognition by top-down and bottom-up processing in cortex: The control of selective attention" Journal of Neurophysiology $90798-810$

Gauthier I, Palmeri T J, 2002 "Visual neurons: Categorization-based selectivity" Current Biology 12 R282-R284

Gauthier I, Tarr M J, 1997 "Orientation priming of novel shapes in the context of viewpointdependent recognition” Perception $2651-73$

Gillebert C R, Op de Beeck H P, Panis S, Wagemans J, 2008 "Subordinate categorization enhances the neural-selectivity in the human object-selective cortex for fine shape differences" Journal of Cognitive Neuroscience, in press

Graf M, 2002 Form, Space and Object: Geometrical Transformations in Object Recognition and Categorization (Berlin: Wissenschaftlicher Verlag Berlin)

Graf M, 2006 "Coordinate transformations in object recognition" Psychological Bulletin 132 $920-945$

Graf M, 2008 "Categorization and object shape", in Understanding Thinking volume II Towards a Theory of Thinking: Structures, Dynamics, Genesis Eds B Glatzeder, A von Müller, V Goel (Berlin: Springer), in press

Graf M, Kaping D, Bülthoff H H, 2005 "Orientation-congruency effects for familiar objects: Coordinate transformations in object recognition" Psychological Science 16 214-221

Hahn U, Chater N, Richardson L B, 2003 "Similarity as transformation" Cognition 87 1-32

Heekeren H R, Marrett S, Bandettini P A, Ungerleider L G, 2004 "A general mechanism for perceptual decision-making in the human brain" Nature 431 859-862

Hernández A, Zainos A, Romo R, 2002 "Temporal evolution of a decision-making process in medial premotor cortex" Neuron 33 959-972

Hillyard S A, Teder-Sälejärvi W A, Münte T F, 1998 "Temporal dynamics of early perceptual processing" Current Opinion in Neurobiology $8202-210$

Hochstein S, Ahissar M, 2002 "View from the top: Hierarchies and reverse hierarchies in the visual system" Neuron $36791-804$

Jäkel F, Schölkopf B, Wichmann F A, 2007 "Generalization and similarity in exemplar models of categorization: Insights from machine learning" Psychonomic Bulletin and Review 15256 - 271

Jolicoeur P, 1990 "Orientation-congruency effects on the identification of disoriented shapes" Journal of Experimental Psychology: Human Perception and Performance 16351 - 364

Kayaert G, Biederman I, Op de Beeck H, Vogels R, 2005 "Tuning for shape dimensions in macaque inferior temporal cortex" European Journal of Neuroscience 22 212-224

Kruschke J K, 1992 "ALCOVE: An exemplar-based connectionist model of category learning" Psychological Review $9922-44$

Kukkonen H T, Foster D H, Wood J R, Wagemans J, Van Gool L, 1996 "Qualitative cues in the discrimination of affine-transformed minimal patterns" Perception 25195 - 206

Lamberts K, 2000 "Information-accumulation theory of speeded categorization" Psychological Review $107227-260$

Larsen A, Bundesen C, 1978 "Size scaling in visual pattern recognition" Journal of Experimental Psychology: Human Perception and Performance $41-20$ 
Larsen A, McIlhagga W, Bundesen C, 1999 "Visual pattern matching: Effects of size ratio, complexity, and similarity in simultaneous and successive matching" Psychological Research $62280-288$

Lawson R, 1999 "Achieving visual object constancy across plane rotation and depth rotation" Acta Psychologica $102221-245$

Lawson R, Humphreys G W, 1998 "View-specific effects of depth rotation and foreshortening on the initial recognition and priming of familiar objects" Perception \& Psychophysics $601052-1066$

Lawson R, Humphreys G W, Jolicoeur P, 2000 "The combined effects of plane disorientation and foreshortening on picture naming: One manipulation or two?" Journal of Experimental Psychology: Human Perception and Performance $26568-581$

Mark L S, Todd J T, 1985 "Describing perceptual information about human growth in terms of geometric invariants" Perception \& Psychophysics $37249-256$

Mark L S, Todd J T, Shaw R E, 1981 "Perception of growth: A geometric analysis of how different styles of change are distinguished" Journal of Experimental Psychology: Human Perception and Performance $7855-868$

Markman A B, Gentner D, 1993a "Structural alignment during similarity comparisons" Cognitive Psychology 25431 - 467

Markman A B, Gentner D, 1993b "Splitting the differences: A structural alignment view of similarity" Journal of Memory and Language 32 517-535

McKeeff T J, Tong F, 2007 "The timing of perceptual decisions for ambiguous face stimuli in the human ventral visual cortex" Cerebral Cortex 17 669-678

Medin D L, Schaffer M M, 1978 "Context theory of classification learning” Psychological Review $85207-238$

Mervis C B, Rosch E, 1981 "Categorization of natural objects" Annual Review of Psychology 32 $89-115$

Mozer M C, 2002 "Frames of reference in unilateral neglect and visual perception: A computational perspective" Psychological Review 109 156-185

Mruczek R E B, Sheinberg D L, 2007 "Activity of inferior temporal cortical neurons predicts recognition choice behavior and recognition time during visual search" Journal of Neuroscience $272825-2836$

Newell F N, Sheppard D M, Edelman S, Shapiro K L, 2005 "The interaction of shape- and location-based priming in object categorization: Evidence for a hybrid 'what + where' representation stage" Vision Research $452065-2080$

Nosofsky R M, 1986 "Attention, similarity, and the identification-categorization relationship" Journal of Experimental Psychology: General $11539-57$

Nosofsky R M, 1988 "Exemplar-based accounts of relations between classification, recognition, and typicality" Journal of Experimental Psychology: Learning, Memory, and Cognition 14 700-708

Nosofsky R M, Palmeri T J, 1997a "An exemplar-based random walk model of speeded classification" Psychological Review $104266-300$

Nosofsky R M, Palmeri T J, 1997b "Comparing exemplar-retrieval and decision-bound models of speeded perceptual classification" Perception \& Psychophysics 59 1027-1048

Nosofsky R M, Zaki S R, 2002 "Exemplar and prototype models revisited: Response strategies, selective attention, and stimulus generalization" Journal of Experimental Psychology: Learning, Memory, and Cognition $28924-940$

Op de Beeck H P, Béatse E, Wagemans J, Sunaert S, Van Hecke P, 2000 "The representation of shape in the context of visual object categorisation tasks" NeuroImage $1228-40$

Op de Beeck H P, Wagemans J, 2001 "Visual object categorization at distinct levels of abstraction: a new stimulus set" Perception $301337-1361$

Op de Beeck H P, Wagemans J, Vogels R, 2001 "Inferotemporal neurons represent low-dimensional configurations of parameterized shapes" Nature Neuroscience $41244-1252$

Op de Beeck H P, Wagemans J, Vogels R, 2003 "Asymmetries in stimulus comparisons by monkey and man" Current Biology $131803-1808$

Op de Beeck H P, Wagemans J, Vogels R, 2008 "The representation of perceived shape similarity and its role for category learning in monkeys: A modeling study" Vision Research $48598-610$

O'Regan J K, 1992 "Solving the 'real' mysteries of visual perception: The world as an outside memory" Canadian Journal of Psychology 46461 - 488

Palmeri T J, Gauthier I, 2004 "Visual object understanding" Nature Reviews Neuroscience 5291 - 304

Palmeri T J, Nosofsky R M, 2001 "Central tendencies, extreme points, and prototype enhancement effects in ill-defined perceptual categorization" Quarterly Journal of Experimental Psychology 54A $197-235$ 
Palmeri T J, Wong A C-N, Gauthier I, 2004 "Computational approaches to the development of perceptual expertise" Trends in Cognitive Sciences $8378-386$

Panis S, De Winter J, Vandekerckhove J, Wagemans J, 2008a "Identification of everyday objects on the basis of fragmented versions of outlines" Perception $37271-289$

Panis S, Vangeneugden J, Op de Beeck H P, Wagemans J, 2008b "The representation of subordinate shape similarity in human occipitotemporal cortex" Journal of Vision 8(10):9, 1-15, http://journalofvision.org/8/10/9, doi:10.1167/8.10.9

Panis S, Wagemans J, 2008 "Time-course contingencies in perceptual organization and identification of fragmented object outlines" Journal of Experimental Psychology: Human Perception and Performance, in press

Perrett D I, Oram M W, Ashbridge E, 1998 "Evidence accumulation in cell populations responsive to faces: an account of generalization of recognition without mental transformations" Cognition $67111-145$

Pittenger J B, Shaw R E, 1975 "Aging faces as viscal elastic events: Implications for a theory of nonrigid shape perception" Journal of Experimental Psychology: Human Perception and Performance $1374-382$

Pittenger J B, Todd J T, 1983 "Perception of growth from changes in body proportions" Journal of Experimental Psychology: Human Perception and Performance 9 945-954

Poggio T, Bizzi E, 2004 "Generalization in vision and motor control" Nature 431 768-774

Rorie A E, Newsome W T, 2005 "A general mechanism for decision-making in the human brain?" Trends in Cognitive Sciences $941-43$

Rybak I A, Gusakova V I, Golovan A V, Podladchikova L N, Shevtsova N A, 1998 "A model of attention-guided visual perception and recognition" Vision Research 38 2387-2400

Salinas E, Sejnowski T, 2001 "Gain modulation in the central nervous system: Where behavior, neurophysiology, and computation meet" Neuroscientist $7430-440$

Schacter D L, 1992 "Priming and multiple memory systems: Perceptual mechanisms of implicit memory" Journal of Cognitive Neuroscience 4 244-256

Schyns P G, 1997 "Categories and percepts: A bi-directional framework for categorization" Trends in Cognitive Sciences $1183-189$

Schyns P G, 1998 "Diagnostic recognition: task constraints, object information, and their interaction" Cognition $67147-179$

Schyns P G, Rodet L, 1997 "Categorization creates functional features" Journal of Experimental Psychology: Learning, Memory, and Cognition $23681-696$

Shepard R N, Cooper L A, 1982 Mental Images and Their Transformations (Cambridge, MA: MIT Press)

Smith J D, Minda J P, 2001 "Journey to the center of the category: The dissociation in amnesia between categorization and recognition" Journal of Experimental Psychology: Learning, Memory, and Cognition $27984-1002$

Snodgrass J G, Vanderwart M, 1980 "A standardized set of 260 pictures: norms for name agreement, image agreement, familiarity, and visual complexity" Journal of Experimental Psychology: Human Learning and Memory 6 174-215

Spivey M J, Dale R, 2006 "Continuous dynamics in real-time cognition" Current Directions in Psychological Sciences 15 207-211

Sugase Y, Yamane S, Ueno S, Kawano K, 1999 "Global and fine information coded by single neurons in the temporal visual cortex" Nature 400 869-873

Tarr M J, 1995 "Rotating objects to recognize them: A case study on the role of viewpoint dependency in the recognition of three-dimensional objects" Psychonomic Bulletin \& Review 2 55-82

Tarr M J, Bülthoff H H, 1998 "Image-based object recognition in man, monkey, and machine" Cognition 67 1-20

Tarr M J, Gauthier I, 1998 "Do viewpoint-dependent mechanisms generalize across members of a class?" Cognition $6773-110$

Tarr M J, Williams P, Hayward W G, Gauthier I, 1998 "Three-dimensional object recognition is viewpoint dependent" Nature Neuroscience $1275-277$

Todd J T, Chen L, Norman J F, 1998 "On the relative salience of Euclidean, affine, and topological structure for 3-D form discrimination" Perception 27273 -282

Trick L M, Enns J T, 1997 "Clusters precede shapes in perceptual organization" Psychological Science $8124-129$

Ullman S, 1989 "Aligning pictorial descriptions: An approach to object recognition" Cognition $32193-254$

Ullman S, 1998 "Three-dimensional object recognition based on the combination of views" Cognition $6721-44$ 
Van Gool L. Moons T, Pauwels E, Wagemans J, 1994 “Invariance from the Euclidean geometer's perspective" Perception $23547-561$

Vanrie J, Béatse E, Wagemans J, Sunaert S, Van Hecke P, 2002 "Mental rotation versus invariant features in object perception from different viewpoints: An fMRI study" Neuropsychologia 40 $917-930$

Vanrie J, Willems B, Wagemans J, 2001 "Multiple routes to object matching from different viewpoints: Mental rotation versus invariant features" Perception $301047-1056$

Vanucci M, Viggiano M P, 2000 "Category effects on the processing of plane-rotated objects" Perception $29287-302$

Vogels R, 1999 "Categorization of complex visual images by rhesus monkeys: Part 2: Single-cell study" European Journal of Neuroscience 11 1239-1255

Vuilleumier P, Henson R N, Driver J, Dolan R J, 2002 "Multiple levels of visual object constancy revealed by event-related fMRI of repetition priming" Nature Neuroscience 5 491-499

Wagemans J, De Winter J, Op de Beeck H, Ploeger A, Beckers T, Vanroose P, 2008 "Identification of everyday objects on the basis of silhouette and outline versions" Perception 37 207-244

Wagemans J, Lamote C, Van Gool L, 1997 "Shape equivalence under perspective and projective transformations" Psychonomic Bulletin \& Review $4248-253$

Wagemans J, Van Gool L, Lamote C, 1996 “The visual system's measurement of invariants need not itself be invariant" Psychological Science $7232-236$

Wagemans J, Van Gool L, Lamote C, Foster D H, 2000 "Minimal information to determine affine shape equivalence" Journal of Experimental Psychology: Human Perception and Performance 26 $443-468$

Weiss Y, Edelman S, 1995 "Representation of similarity as a goal of early visual processing" Computation in Neural Systems $619-41$

Wiggs C L, Martin A, 1998 "Properties and mechanisms of perceptual priming" Current Opinion in Neurobiology $8227-233$

Willems B, Wagemans J, 2001 "Matching multi-component objects from different viewpoints: Mental rotation as normalization?" Journal of Experimental Psychology: Human Perception and Performance 27 1090-1115

Wyss R, König P, Verschure P F M J, 2003 "Invariant representations of visual patterns in a temporal population code" Proceedings of the National Academy of Sciences of the USA 100 $324-329$

Zaki S R, Nosofsky R M, Stanton R D, Cohen A L, 2003 "Prototype and exemplar accounts of category learning and attentional allocation: A reassessment” Journal of Experimental Psychology: Learning, Memory, and Cognition $291160-1173$ 


\section{PERTEPTION}

VOLUME 372008

www.perceptionweb.com

Conditions of use. This article may be downloaded from the Perception website for personal research by members of subscribing organisations. Authors are entitled to distribute their own article (in printed form or by e-mail) to up to 50 people. This PDF may not be placed on any website (or other online distribution system) without permission of the publisher. 